\title{
Quantitative comparison of reconstruction methods for intra-voxel fiber recovery from diffusion MRI
}

\author{
Alessandro Daducci*, Erick Jorge Canales-Rodríguez, Maxime Descoteaux, Eleftherios Garyfallidis, Yaniv Gur, \\ Ying-Chia Lin, Merry Mani, Sylvain Merlet, Michael Paquette, Alonso Ramirez-Manzanares, Marco Reisert, \\ Paulo Reis Rodrigues, Farshid Sepehrband, Emmanuel Caruyer, Jeiran Choupan, Rachid Deriche, Mathews \\ Jacob, Member, IEEE, Gloria Menegaz, Vesna Prčkovska, Mariano Rivera, Yves Wiaux, Member, IEEE, \\ Jean-Philippe Thiran, Senior Member, IEEE
}

\begin{abstract}
Validation is arguably the bottleneck in the diffusion MRI community. This paper evaluates and compares 20 algorithms for recovering the local intra-voxel fiber structure from diffusion MRI data and is based on the results of the "HARDI reconstruction challenge" organized in the context of the "ISBI 2012" conference. Evaluated methods encompass a mixture of classical techniques well-known in the literature such as Diffusion Tensor, Q-Ball and Diffusion Spectrum imaging, algorithms inspired by the recent theory of compressed sensing and also brand new approaches proposed for the first time at this contest. To quantitatively compare the methods under controlled conditions, two datasets with known ground-truth were synthetically generated and two main criteria were used to evaluate the quality of the reconstructions in every voxel: correct assessment of the number of fiber populations and angular accuracy in their orientation. This comparative study investigates the behavior of every algorithm with varying experimental conditions and highlights strengths and weaknesses of each approach.
\end{abstract}

A. Daducci is with the Signal Processing Lab (LTS5), École Polytechnique Fédérale de Lausanne (EPFL), Switzerland

E.J. Canales-Rodríguez is with the FIDMAG Germanes Hospitaláries, Spain, and also with the Centro de Investigación Biomédica en Red de Salud Mental, CIBERSAM, Spain

M. Descoteaux and M. Paquette are with the Sherbrooke Connectivity Imaging Lab (SCIL), Université de Sherbrooke, Canada

E. Garyfallidis is with the Wolfson College, University of Cambridge, UK, and also with the Cognition and Brain Sciences Unit, MRC, UK

Y. Gur is with the SCI Institute, University of Utah, USA

Y.-C. Lin and G. Menegaz are with the department of Computer Science, University of Verona, Italy

M. Mani and M. Jacob are with the department of Electrical Engineering, University of Rochester, USA

S. Merlet, E. Caruyer and R. Deriche are with the Athena Project-Team, INRIA Sophia Antipolis - Méditerranée, France

A. Ramirez-Manzanares is with the department of Mathematics, University of Guanajuato, Mexico

M. Reisert is with the Medical Physics, department of Radiology, University of Freiburg Medical Center, Germany

P.R. Rodrigues is with the department de Personalitat, Avaluacioó i Tr. Psicoloógics, Facultat de Psicologia, Universitat de Barcelona, Spain

F. Sepehrband and J. Choupan are with the Centre for Advanced Imaging, The University of Queensland, Australia

V. Prčkovska is with the department of Neuroimmunology, Institut d'investigacions Biomédiques August Pi i Sunyer (IDIBAPS), Spain

M. Rivera is with the Centro de Investigación en Matemáticas A.C. (CIMAT), Mexico

Y. Wiaux is with the Signal Processing Lab (LTS5), École Polytechnique Fédérale de Lausanne (EPFL), Switzerland, and with the department of Radiology and Medical Informatics, University of Geneva, Switzerland, and also with the Institute of Sensors, Signals \& Systems, Heriot-Watt University, Edinburgh EH14 4AS, UK

J.-P. Thiran is with the Signal Processing Lab (LTS5), École Polytechnique Fédérale de Lausanne (EPFL), Switzerland, and also with the University Hospital Center (CHUV) and University of Lausanne (UNIL), Switzerland

$*$ indicates the corresponding author
This information can be useful not only for enhancing current algorithms and develop the next generation of reconstruction methods, but also to assist physicians in the choice of the most adequate technique for their studies.

Index Terms-diffusion MRI, local reconstruction, quantitative comparison, validation, synthetic data

\section{INTRODUCTION}

D IFFUSION MRI (dMRI) is a powerful magnetic resonance imaging modality which is sensitive to the Brownian motion of water molecules in living tissues. This peculiar feature is exceptionally effective in the brain as it permits to investigate the structural wiring of the nervous system in vivo and non-invasively. In fact, in the white matter, these microscopic random movements are highly hindered by the coherent organization in bundles of the neuronal tissue [1] and it is possible to infer the architecture of the neuronal connections by properly characterizing the distribution of the displacements. The richer and more detailed this description is, the more accurate any subsequent global connectivity analysis will be. In fact, all fiber-tracking algorithms [2], [3] (see [4] and references therein for a more complete list) rely on a suitable characterization of the diffusion process within each voxel as they attempt to estimate the most likely fiber tracts connecting different regions of the brain.

The first approach to model the anisotropic nature of the diffusion process in biological tissues has been Diffusion Tensor Imaging [5] (DTI), which is based on the Gaussian assumption on the displacements. Despite providing useful diagnostic information with very fast acquisitions, DTI is unable to resolve multiple fiber populations within a voxel. To overcome this limitation, Diffusion Spectrum Imaging [6] (DSI) has been proposed. DSI is a model-free technique exclusively based on the Fourier relationship [7] between the signal acquired in a Cartesian grid and the 3D displacement spectrum, also known as the Ensemble Average Propagator (EAP). DSI provides probably the most detailed description of diffusion within each voxel, but it requires hundreds of measurements which are not compatible with the normal acquisition time admitted in clinical protocols. To this aim, a large number of alternative reconstruction techniques have been developed to recover both the angular and radial component of the diffusion process. Among them, to mention a few, we can cite the models based on multiple [8]-[11] or higher-order tensors [12]-[14], 
the Q-Ball Imaging (QBI) [15] and its variant computed in constant solid angle [16], [17], the Persistent Angular Structure MRI (PAS-MRI) [18], the Composite Hindered And Restricted Model of Diffusion (CHARMED) [19], the Diffusion Orientation Transform (DOT) [20], [21], the Hybrid Diffusion Imaging (HIDY) [22], the Generalized Q-sampling Imaging (GQI) [23] and all the algorithms based on spherical deconvolution [24]-[29]. Multi-compartment models have been also proposed in the literature to explain the measured dMRI signal more accurately; see [30] for a detailed survey. All these techniques are characterized by fitting advanced and more descriptive models of diffusion (e.g. intra- and extra-axonal space) to get access to important micro-structure parameters such as axonal diameter and density. Lately, the advent of compressed sensing [31], [32] inspired new approaches to solve the reconstruction problem whose main goal is to drastically reduce the acquisition time [33]-[39]. This list is not exhaustive and many other algorithms exist in the literature; see [40]-[42] for a more comprehensive list of available techniques. All these methods differ in a great deal of aspects, like for instance the acquisition scheme adopted (number and position of the diffusion gradients), the targeted feature of the diffusion process to estimate, e.g. EAP, diffusion Orientation Distribution Function (ODF) or Fiber Orientation Distribution (FOD), and the assumptions made in the reconstruction. A direct comparison among techniques is hence very difficult.

Another source of confusion when comparing the approaches proposed in the literature stems from the different choices of the framework used to validate the reconstructions. Validation frameworks can be divided into three categories: biological phantoms, physical phantoms and numerical simulations. In the first case, the fiber configurations estimated from dMRI are compared with the results of histological or tracerinjection studies [43]-[46], either in vivo or ex vivo, performed on the same biological sample. In particular, an emerging technique called three-dimensional Polarized Light Imaging (3D-PLI) [47] is capable of providing the directionality of the axons in postmortem human brains at ultra-high resolution, i.e. sub-millimeter scale. All these techniques offer exquisite insights of the brain structure but they are very invasive and, more importantly, allow to investigate only a few specific axonal tracts or sample slices at once. On the other hand, with physical phantoms [48]-[57] it is possible to build specific arrangements of synthetic fibers that mimic bending, crossing and kissing configurations usually observed in the real brain anatomy and thus validate the algorithms in more complex scenarios. However, the construction of a realistic phantom is an extremely challenging task as the dimension and the complexity of the fiber architecture which is possible to achieve with current technologies are orders of magnitude different from the real nervous system. Moreover, all publicly available phantoms have normally been scanned only a limited number of times, hence providing images corresponding to a very limited number of sampling schemes and with only an intrinsic acquisition noise. Therefore they are not suitable for testing the performances of an algorithm in different scenarios. By contrast with the two previous validation frameworks, numerical simulations offer a simple and versatile way to assess the performances of a reconstruction algorithm with a broader set of experimental conditions, e.g. signal models [8], [58], fiber configurations, sampling schemes and noise levels. Interestingly, Tournier et al. [48] have shown that numerical simulations performed using these models agree well with physical phantoms but, as already mentioned, they represent an over-simplification of what happens in the white matter. Even though the level of complexity they offer is way too simplistic with respect to the real architecture of the nervous system, they actually constitute the validation framework of choice for the majority of the algorithms proposed in the literature, as in [8], [17], [20], [23], [27], [28], [33], [34], [37], [59], [60] to mention a few. Unfortunately, when a new algorithm is proposed, the performances are normally assessed with ad-hoc synthetic data and evaluation criteria, and comparing different approaches can be difficult. This lack of consensus on the validation framework is particularly crucial in a clinical perspective, as the availability of a comprehensive comparison of the most common techniques might help clinicians in the choice of the most adequate method for a specific application.

With these considerations in mind, the aim of this work is to provide all researchers in this field with a common validation framework to assess the performances of their own algorithms for the intra-voxel fiber recovery and fairly compare their results against other approaches under controlled conditions. It is not an intention of this work to determine the most suitable technique to adopt in a clinical study, as the choice highly depends both on the interests (e.g. research question or intravoxel feature to extract) and possibilities (e.g. available scan time) of the study itself. Nor it is likely that an algorithm outperforms all the others in all possible scenarios. This study should be intended as a comprehensive set of analyses reporting strengths and weaknesses of a large selection of state-ofthe-art techniques in a wide range of experimental conditions. The focus is on the angular component of the recovered diffusion profiles. Because of the large number of techniques proposed in the literature to this end, the evaluation presented in this work is not exhaustive. Nonetheless, to date and to the best of our knowledge it represents the comparison study with the largest number of different reconstruction techniques and the most heterogeneous set of experimental conditions.

The rest of the manuscript is organized as follows. In section II we present the challenge, describing in detail its organization, the ground-truth synthetic data and the evaluation criteria used, and the reconstruction methods that were considered in the study. The results of the comparison are presented and discussed in section III. We conclude this paper with general considerations and some take-home messages.

\section{MATERiAls AND METHODS}

In this section we describe in detail the organization of the contest, the reconstruction methods considered in this study and the evaluation framework that we used to quantitatively compare all the algorithms, i.e. synthetic datasets with known ground-truth and evaluation criteria. More information can be found on the challenge website ${ }^{1}$.

\footnotetext{
${ }^{1}$ hardi.epfl.ch/static/events/2012_ISBI
} 


\section{A. Contest organization}

The diffusion MRI reconstruction challenge was organized in the context of the $9^{\text {th }}$ IEEE International Symposium on Biomedical Imaging (ISBI) conference, which was held in Barcelona (Spain) in May 2012. The purpose of this contest was to evaluate and compare different reconstruction methods for recovering the intra-voxel fiber structure, i.e. number and orientation of the fibers populations present in each voxel, from dMRI acquisitions, using the same ground-truth data and under controlled conditions.

Training data was released prior to the contest in order for the contestants to practice with the data format, test their methods and identify the setting providing the best results, e.g. acquisition protocol and specific parameters of the algorithm. For the final evaluation of the performances of each method, though, another set of testing data was used. In order to grant the maximum flexibility for the acquisition scheme required by each reconstruction algorithm, while still keeping the groundtruth of the testing data unknown to the contestants, we adopted the following single-blind procedure for the generation of the dMRI signal. Each participant had the possibility to probe the signal corresponding to the ground-truth dataset only once by submitting to the organizers an acquisition scheme specifying the sampling coordinates in q-space, i.e. direction and strength of the diffusion gradients. The minimum and maximum number of samples which was possible to probe was fixed to 6 and 257, respectively, corresponding to standard DTI and DSI acquisitions on one hemisphere, assuming central symmetry in the diffusion signal. There were no restrictions, however, on the position of the samples in the q-space.

The signal was then numerically simulated in every voxel of the dataset by the organizers (see next section) according to the gradient list received from each team. This is the only information the contestants knew about the common dataset. For teams participating with more than one method, data sharing between the algorithms was not allowed. At this point, the participants could apply their proposed reconstruction algorithms on the data received from the organizers in order to recover the underlying fiber configuration. Any combination of algorithms, signal processing techniques and available software was allowed, with the only constraint that the outcomes of the proposed methodology must be reproducible.

\section{B. Synthetic data}

1) Signal simulation: To simulate the dMRI signal intensity in a voxel with $M$ fiber populations and corresponding to the application of a diffusion-sensitizing gradient we adopted the classical Gaussian Mixture Model proposed by [8]:

$$
S(\mathbf{q})=S_{0} \sum_{i=1}^{M} f_{i} \exp \left(-b \mathbf{u}^{T} \mathbf{D}_{(i)} \mathbf{u}\right)
$$

where $\mathbf{q}$ is the coordinate vector in $\mathbf{q}$-space accounting for the direction and strength of the diffusion gradient, $\mathbf{u} \in \mathrm{S}^{2}$ is a unit vector in $3 \mathrm{D}$ with $\mathbf{q}=q \mathbf{u}=|\mathbf{q}| \mathbf{u}, b=4 \pi^{2} q^{2} t$ is the b-value corresponding to $q$ and diffusion time $t$ and $S_{0}$ is the signal without diffusion weighting (i.e. $b=0$ ). Each fiber population can be characterized by a tensor $\mathbf{D}_{(i)}$ and a non-negative volume fraction $f_{i}$ such that $\sum_{i=1}^{M} f_{i}=1$. Each $\mathbf{D}_{(i)}$ can be expressed as a rotated version of a tensor $\mathbf{D}=\operatorname{diag}\left(\lambda_{1}, \lambda_{2}, \lambda_{3}\right)$ as $R^{T} \mathbf{D} R, R$ being the rotation matrix rotating the main axis of $\mathbf{D}$ to the direction of a given fiber population. Diagonal elements of $\mathbf{D}$ are the diffusivities along the main axis of the fiber $\left(\lambda_{1}\right)$ and in the plane perpendicular to it $\left(\lambda_{2}, \lambda_{3}\right)$. In this contest, the diffusivities were generated from the following ranges typically observed in the human brain [60]: $\lambda_{1} \in[1,2] \times 10^{-3} \mathrm{~mm}^{2} / \mathrm{s}$ and $\lambda_{2}=\lambda_{3} \in[0.1,0.6] \times 10^{-3} \mathrm{~mm}^{2} / \mathrm{s}$. Also, we assumed $S_{0}=1$ without loss of generality.

2) Noise simulation: To be consistent with previous studies presenting new reconstruction methods, in this work the signal $S$ has been corrupted by Rician noise [61] as follows:

$$
S_{\text {noisy }}=\sqrt{\left(S+\eta_{1}\right)^{2}+\eta_{2}^{2}}
$$

with $\eta_{1}, \eta_{2} \sim \mathcal{N}\left(0, \sigma^{2}\right)$ and $\sigma=S_{0} / S N R$ controls the level of the noise, which is reported here as the signal-to-noise ratio (SNR) on the $S_{0}$ image. In our simulations the only source of artifact is Rician noise and the performances of the reconstruction methods have been evaluated as function of its magnitude for three different noise levels, i.e. $\mathrm{SNR}=10,20$, 30. The study of the quality of the reconstructions as function of other realistic imaging artifacts such as eddy currents, $B_{0}$-field inhomogeneity and subject motion, was beyond the scope of this work.

3) Phantoms: In order to adequately evaluate the performances of each algorithm with different experimental conditions we created two separate synthetic phantoms.

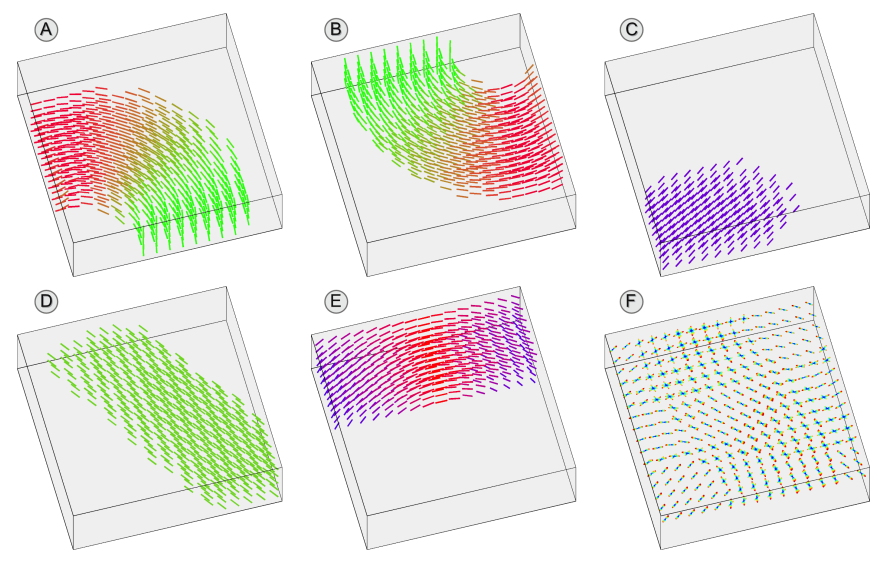

Fig. 1. Structured field synthetic dataset. The 5 different fiber bundles (A-E) are reported separately. In each voxel, the directions of the fiber populations are color-coded depending on their orientation (x-axis, y-axis, $\mathrm{Z}$-axis). Subplot $\mathrm{F}$ shows a representative slice with the corresponding ODFs computed analytically from the equation (1) as in [15], [60].

The structured field dataset (hereafter $\mathrm{SF}$ ) consists of a $16 \times 16 \times 5$ volume attempting to simulate a realistic $3 \mathrm{D}$ configuration of tracts occurring in reality. As shown in Fig. 1, this dataset comprises five different fiber bundles which give rise to non-planar configurations of bending, crossing and kissing tracts. All fiber tracts are characterized by having a fractional anisotropy between 0.75 and 0.90 . The diffusion properties have been generated from the diffusivity ranges previously described and were kept constant along the full 
trajectory of each bundle. The dataset contains the following proportion of voxel configurations: $35 \%$ with a single fiber population, $35 \%$ with two and $30 \%$ with three. In voxels with multiple fiber populations, then, the crossing angles between the fiber directions were distributed as follows: $27 \%$ smaller than $30^{\circ}, 35 \%$ in the range $31^{\circ}-60^{\circ}$ and $38 \%$ above $61^{\circ}$. The purpose of this dataset was to test the average performance of each algorithm with plausible fiber configurations. Moreover, we used this dataset to investigate the ability of each algorithm to exploit additional information from neighboring voxels for possibly improving the reconstruction of the fiber configuration at a given position.

On the other hand, the isolated voxels dataset (hereafter IV) consists of a set of 9100 independent voxels with no spatial coherence among them. Each voxel contains a given configuration of 2 fiber populations crossing at specific angles, with 100 repetitions of every given configuration. The diffusion properties of each population have been randomly generated by means of uniform distributions in the ranges previously described, but the two fiber populations do not necessarily share the same values. As for the SF dataset, the fractional anisotropy was maintained between 0.75 and 0.90 . For completeness, the analysis of the performance of each reconstruction algorithm is provided in the full range of crossing angles, $0^{\circ}-90^{\circ}$, as in [17], [38], [48]. The purpose of this dataset is to explore more in detail the behavior of each approach as some experimental conditions change.

\section{Evaluation criteria}

To assess the quality of the reconstructions we have focused our attention on two main criteria: (i) correct assessment of the number of fiber populations present in each voxel and (ii) angular accuracy in their orientation. We have purposely adopted evaluation metrics widely used in the literature, e.g. [26], [29], [36]-[38], [62], [63], with the aim to simplify and standardize the comparison of our results with previously reported findings. To compute these metrics we have compared the fiber populations (also referred to as fiber compartments in this work) of the ground-truth with the directions estimated by each reconstruction algorithm. There was not consensus, though, in the strategy to estimate these directions: some teams used peak-finding procedures on the recovered ODFs while others extracted them directly in the reconstruction problem (e.g. DTI), some used a threshold to filter out small peaks and some other did not. More information on each method's strategy can be found on the challenge website.

In order to assess the correct estimation of the number of fiber compartments we employed the success rate, hereafter reported as $S R$, which is defined as the proportion of voxels in which a reconstruction algorithm estimates correctly the right number of fiber populations. To understand the reasons of an incorrect assessment, we also used the following two quantities, $n^{+}$and $n^{-}$, which explicitly count the number of over-estimated and under-estimated fiber populations in a voxel, respectively. Furthermore, in order to distinguish between the estimated fiber populations that are close enough to the real ones, i.e. true positives, and those which are clearly spurious, i.e. false positives, we recomputed the previous metrics using a tolerance cone as suggested in [26], [63]. These three additional measures, namely $S R_{L}, n_{\angle}^{+}$and $n_{\angle}^{-}$, are defined as before but an estimated direction is considered "resolved" only if it falls within a tolerance cone around a real fiber population in the voxel. In this work the tolerance was set to $20^{\circ}$. Noteworthy, in this study we have employed both versions of these metrics, i.e. with and without tolerance cone, as this provided more insight into the reconstructions.

The angular accuracy in the orientation of the estimated fiber compartments was assessed by means of the average error (in degree) between the estimated fiber directions and the true ones present in a voxel:

$$
\bar{\theta}=\frac{180}{\pi} \arccos \left(\left|\mathbf{d}_{\text {true }} \cdot \mathbf{d}_{\text {estimated }}\right|\right),
$$

where the unitary vectors $\mathbf{d}_{\text {true }}$ and $\mathbf{d}_{\text {estimated }}$ are, respectively, a true fiber population in the voxel and the closest of the estimated directions; then - denotes the dot product. The final value in the voxel is the average computed by first matching the directions estimated by the algorithm to the ground-truth in order to minimize the error.

\section{Reconstruction methods}

Table I gives an overview of the 20 algorithms considered in this work. For more details about each reconstruction method (not reported here for conciseness), please refer to the proceedings of the workshop on the challenge website. In addition to the 13 submissions received during the contest, in this study we also considered 7 additional well-established reconstruction algorithms with the aim to have a more comprehensive comparison. To prevent unfair comparisons among methods using so different acquisition protocols, the algorithms have been grouped into four different classes depending on the characteristics of the sampling scheme adopted.

The DTI-like class includes approaches based on clinical acquisition protocols with less than 15 samples and maximum $\mathrm{b}$-value equal to $1000 \mathrm{~s} / \mathrm{mm}^{2}$. In addition to the classical Diffusion Tensor Imaging [5] (DTI), this class comprises a novel approach named $\mathrm{DTI}_{\text {neigh }}$ [64] which aims at improving the reconstructions by means of contextual enhancement of the spherical diffusion functions based on the notion of context.

In the HARDI-like class we grouped those methods based on acquisition schemes with one or multiple shells using a maximum b-value of $3000 \mathrm{~s} / \mathrm{mm}^{2}$. Six well established techniques have been included: Diffusion Orientation Transform [20] (DOT), analytical Q-Ball Imaging [59] (QBI), Q-Ball Imaging with Spherical Wavelet Transform [66] (QBI ${ }_{\text {SWT }}$ ), QBall Imaging in Constant Solid Angle [17] $\left(\mathrm{QBI}_{\mathrm{CSA}}\right)$, Nonnegativity Constrained Super-resolved Spherical Deconvolution [27] (CSD) and the Sharpening Deconvolution Transform [29] (SDT). In addition, two novel algorithms have been considered: STD, a spherical deconvolution approach based on Symmetric Tensor Decomposition, and SPIRAL, an innovative technique based on spiral sampling which exploits the periodic pattern of the signal in the gradient direction domain for denoising and estimating the intra-voxel fiber configuration. 
TABLE I

SUMMARY OF THE RECONSTRUCTION ALGORITHMS EVALUATED IN THIS STUDY. THE METHODS HAVE BEEN GROUPED INTO FOUR DIFFERENT CLASSES, DEPENDING ON THE SAMPLING SCHEME ADOPTED: DTI-like (VIOLET), SPARSE-like (GREEN), HARDI-like (YELLOW-RED) AND DSI-like (BLUE).

THE COLORS USED HERE WILL BE USED COHERENTLY THROUGHOUT THE MANUSCRIPT TO IDENTIFY EACH ALGORITHM.

\begin{tabular}{|c|c|c|c|}
\hline METHOD & ACQUISITION & BRIEF DESCRIPTION OF THE ALGORITHM & SPATIAL FILTER \\
\hline DTI & $\begin{array}{l}\text { one-shell } \\
b=1000 \\
6 \text { samples }\end{array}$ & Diffusion Tensor Imaging (DTI) [5] & none \\
\hline $\mathrm{DTI}_{\text {neigh }}$ & $\begin{array}{c}\text { one-shell } \\
b=1000 \\
12 \text { samples }\end{array}$ & $\begin{array}{l}\text { Contextual enhancing of spherical diffusion functions for improving DTI recon- } \\
\text { structions, based on the notion of context to extrapolate additional information } \\
\text { about the underlying fiber structure [64] }\end{array}$ & regularization \\
\hline L2-L1-DL & $\begin{array}{l}\text { one-shell } \\
b=2000 \\
15 \text { samples }\end{array}$ & $\begin{array}{l}\text { A parametric dictionary is learnt from a set of training diffusion data, providing } \\
\text { a highly sparse representation of the diffusion signal and enabling to analytically } \\
\text { recover important diffusion features as the ODF with a small number of measure- } \\
\text { ments [39] }\end{array}$ & none \\
\hline L1-L1 & $\begin{array}{c}\text { one-shell } \\
b=750 \\
24 \text { samples }\end{array}$ & $\begin{array}{l}\text { Sparse reconstruction using the } \ell_{1} \text { norm both in the data fidelity term and the } \\
\text { sparsity regularization with a Gaussian diffusion profile dictionary }\end{array}$ & none \\
\hline L2-L1-TV & $\begin{array}{l}\text { one-shell } \\
b=1200 \\
30 \text { samples }\end{array}$ & $\begin{array}{l}\text { Sparse reconstruction with combined q-space and k-space with Total Variation } \\
\text { regularization in the spatial domain [65] }\end{array}$ & regularization \\
\hline L2-L2 & $\begin{array}{l}\text { one-shell } \\
b=3333 \\
37 \text { samples }\end{array}$ & $\begin{array}{l}\text { Multi-tensor fitting guided by ODF estimation, to obtain the true diffusivities } \\
\text { corresponding to each different fiber population in the voxel. In the estimation } \\
\text { process, the number, orientations and relative intensities of fiber populations were } \\
\text { assumed to be known and equal to the values determined from the ODF maxima } \\
\text { computed as in [21] }\end{array}$ & smoothing \\
\hline NN-L2 & $\begin{array}{c}\text { one-shell } \\
b=1500 \\
48 \text { samples }\end{array}$ & $\begin{array}{l}\text { Diffusion Basis Functions + selective spatial smoothing based on FA and diffusion } \\
\text { orientation similarity [33] }\end{array}$ & smoothing \\
\hline L2-L1-TGV & $\begin{array}{l}\text { multi-shell } \\
b \in\{1500,2500\} \\
64 \text { samples }\end{array}$ & $\begin{array}{l}\ell_{1} \text {-based constrained spherical deconvolution with Total Generalized Variation } \\
\text { regularization optimized by a first-order primal-dual approach }\end{array}$ & regularization \\
\hline DOT & $\begin{array}{c}\text { one-shell } \\
b=3000 \\
60 \text { samples }\end{array}$ & $\begin{array}{l}\text { Diffusion Orientation Transform (DOT) [20] with the following parameters: Spher- } \\
\text { ical Harmonics (SH) order } \ell_{\max }=8 \text { and EAP evaluated at radius } R=15 \mu \mathrm{m}\end{array}$ & none \\
\hline QBI & $\begin{array}{l}\text { one-shell } \\
b=3000 \\
60 \text { samples }\end{array}$ & $\begin{array}{l}\text { Analytical Q-Ball Imaging (QBI) [59] with the following parameters: } \mathrm{SH} \text { order } \\
\ell_{\max }=6 \text { and regularization parameter } \lambda=0.006\end{array}$ & none \\
\hline $\mathrm{QBI}_{\mathrm{SWT}}$ & $\begin{array}{l}\text { one-shell } \\
b=3000 \\
60 \text { samples }\end{array}$ & $\begin{array}{l}\text { Q-Ball imaging with Spherical Wavelet Transform [66] with the following param- } \\
\text { eters: SH order } \ell_{\max }=8\end{array}$ & none \\
\hline $\mathrm{QBI}_{\mathrm{CSA}}$ & $\begin{array}{l}\text { one-shell } \\
b=3000 \\
60 \text { samples }\end{array}$ & $\begin{array}{l}\text { Q-Ball imaging in Constant Solid Angle [17] with the following parameters: SH } \\
\text { order } \ell_{\max }=6 \text { and regularization parameter } \lambda=0.006\end{array}$ & none \\
\hline CSD & $\begin{array}{l}\text { one-shell } \\
b=3000 \\
60 \text { samples }\end{array}$ & $\begin{array}{l}\text { Non-negativity constrained super-resolved spherical deconvolution (CSD) [27] } \\
\text { with the following parameters: SH order } \ell_{\max }=8 \text { and diffusion profile of } \\
{[1.7,0.3,0.3] \times 10^{-3} \mathrm{~mm}^{2} / \mathrm{s}}\end{array}$ & none \\
\hline SDT & $\begin{array}{l}\text { one-shell } \\
b=3000 \\
60 \text { samples }\end{array}$ & $\begin{array}{l}\text { Sharpening Deconvolution Transform [29] with the following parameters: SH order } \\
\ell_{\max }=6 \text { and diffusion profile of }[1.7,0.3,0.3] \times 10^{-3} \mathrm{~mm}^{2} / \mathrm{s}\end{array}$ & none \\
\hline STD & $\begin{array}{l}\text { one-shell } \\
b=3000 \\
64 \text { samples }\end{array}$ & Spherical deconvolution via Symmetric Tensor Decomposition [67] & none \\
\hline SPIRAL & $\begin{array}{c}\text { spiral } \\
b=1200 \\
82 \text { samples }\end{array}$ & $\begin{array}{l}\text { Gradient directions were set on a spherical spiral curve resulting in voxel-wise } \\
\text { diffusion signal periodicity. The periodic pattern of the signal in the gradient } \\
\text { direction domain was exploited for de-noising and fiber orientation estimation }\end{array}$ & none \\
\hline DSI & $\begin{array}{c}\text { cartesian } \\
b \leq 8000 \\
257 \text { samples }\end{array}$ & $\begin{array}{l}\text { Diffusion Spectrum Imaging (DSI) [6] with the following parameters: zero-padded } \\
35 \times 35 \times 35 \text { grid for the EAP reconstruction, Hanning filtering before discrete } \\
\text { Fourier transformation and ODF integration over the full radius range }\end{array}$ & none \\
\hline $\mathrm{DSI}_{\mathrm{LR}}$ & $\begin{array}{c}\text { cartesian } \\
b \leq 8000 \\
257 \text { samples }\end{array}$ & $\begin{array}{l}\text { General framework based on Lucy-Richardson deconvolution to rectify the EAP } \\
\text { reconstructed with DSI, taking into account the blurring introduced in the Fourier } \\
\text { inversion due to the discrete and finite support of q-space measurements [68] }\end{array}$ & none \\
\hline $\mathrm{DSI}_{\mathrm{SWT}}$ & $\begin{array}{c}\text { cartesian } \\
b \leq 8000 \\
257 \text { samples }\end{array}$ & $\begin{array}{l}\text { Multi-resolution approach based on 3D Stationary Wavelet Transform (3D-SWT) } \\
\text { to de-noise the propagator reconstructed with DSI }\end{array}$ & none \\
\hline $\mathrm{GQI}_{2}$ & $\begin{array}{c}\text { cartesian } \\
b \leq 8000 \\
257 \text { samples }\end{array}$ & $\begin{array}{l}\text { Extension of the Generalized Q-sampling Imaging [23] to analytically reconstruct } \\
\text { the ODF with solid angle consideration. As it is expressed as a direct linear } \\
\text { transform of the raw signal, it is faster to compute than DSI }\end{array}$ & none \\
\hline
\end{tabular}


The DSI-like class encompasses reconstruction methods based on q-space Cartesian sampling and maximum b-value of $8000 \mathrm{~s} / \mathrm{mm}^{2}$. The standard Diffusion Spectrum Imaging [6] (DSI) is studied in comparison to three additional methods that aim at improving its known weaknesses. First, the method $\mathrm{DSI}_{\mathrm{LR}}$ [68] employs the Lucy-Richardson deconvolution to rectify the EAP reconstructed taking into account the blurring effect introduced in the Fourier inversion by standard DSI due to the discrete and finite support of q-space measurements. The second algorithm, $\mathrm{DSI}_{\mathrm{SWT}}$, is a multi-resolution approach based on the 3D Stationary Wavelet Transform (3D-SWT) whose aim is de-noising the propagator reconstructed with DSI to obtain sharper ODFs. At last, GQI 2 extends the Generalized Q-sampling Imaging [23] to analytically reconstruct the ODF with solid angle consideration. Furthermore, as $\mathrm{GQI}_{2}$ is formulated as a efficient linear transform of the raw signal, it is sensibly faster than standard DSI to reconstruct the EAP and, consequently, the ODF.

In the last class, that we term SPARSE-like, we have evaluated six different dictionary-based reconstruction approaches inspired by the recent theory of compressed sensing [31], [32]. These methods are generally formulated as convex optimization problems and exploit various sorts of priors. NN-L2 [33] and L2-L2 [21], for example, represent two extensions to already published works and the corresponding formulations are based on $\ell_{2}$ norm priors. On the other hand, the remaining four algorithms are all based on the $\ell_{1}$ norm for promoting sparsity. Notably, these approaches were proposed for the first time at the contest. L1-L1 is based on a Gaussian dictionary estimated directly from the acquired diffusion data and exploits the $\ell_{1}$ norm both in the data and the sparsity regularization terms; this $\ell_{1}-\ell_{1}$ formulation had never been used in local diffusion estimation before. L2-L1-DL uses the more common $\ell_{2}-\ell_{1}$ paradigm of the reconstruction problem [31], [32], but implements an efficient strategy to learn a parametric dictionary from a set of training data. Such estimated dictionary enables highly sparse representations of the diffusion signal and allows to analytically estimate important diffusion features, such as EAP and ODF, with a very limited number of measurements [39]. The last two approaches, L2-L1-TV [65] and L2-L1-TGV, both use the same $\ell_{2}-\ell_{1}$ problem formulation but they implement two different spatial regularization schemes based on Total Variation and Total Generalized Variation, respectively. It should be noted that in case of the IV dataset, for which no spatial coherence exists among voxels, these two algorithms do not make use of these spatial regularization schemes. In this scenario they can be considered two different implementations, but conceptually equivalent, of a reconstruction algorithm based on the classical $\ell_{2}-\ell_{1}$ paradigm and no spatial regularization, as for instance [34], [38]. Hence, we can assume that we have also evaluated in this comparison, for free, an additional reconstruction algorithm, which might be called L2-L1 for consistency, whose performances can always be assumed equivalent to those of L2-L1-TV and L2-L1-TGV on the IV phantom.

In contrast to many previous studies, the comparison provided in this work is as fair as possible as the majority of the reconstructions were tested using the original implemen- tations provided by their own developers. We used in-house implementations for the additional 7 classical techniques we evaluated, with the exception of DTI and CSD whose reconstructions were computed using FSL [9] and MRTRIX [69], respectively.

As last remark, we point out a few notes concerning some algorithms and their corresponding results. As the method DTI $I_{\text {neigh }}$ needs to have access to the neighborhood of a voxel to work properly, only the reconstructions relative to the $\mathrm{SF}$ dataset were submitted. For this reason, this algorithm was evaluated only for this phantom whereas its performances on the IV dataset can be assumed, as a reference, as good as those of DTI. On the other hand, the method SPIRAL internally fixed a priori the number of directions to extract and it always reconstructed two fibers. As this inevitably biases all the corresponding evaluations, the reader should keep in mind this fact throughout the manuscript.

\section{RESUlts AND Discussion}

The reader is recommended to keep the Table I at his fingertips while examining the following results. In fact, two methods might have obtained similar results but using radically different acquisitions schemes, which makes a direct comparison very difficult. To this aim, as already said, the algorithms have been grouped into four separate sampling classes and, to further simplify the reading, are listed in Table I and in all the plots with increasing number of samples.

\section{A. Overall reconstruction quality}

To provide the reader with a first impression of the algorithms with plausible fiber configurations likely to happen in real human brain data, we assessed the overall quality of the reconstructions in every voxel of the SF dataset in a scenario with a mild level of noise. Fig. 2 reports the summary of the two metrics $S R$ and $\bar{\theta}$ corresponding to the simulations performed with a $\mathrm{SNR}=30$. The robustness to noise of each algorithm will be investigated in detail later in the paper.

The first result to meet the eye is the expected inability of DTI to distinguish multiple fiber populations in a voxel, as clearly reflected in the lowest $S R$ among all methods and high $\bar{\theta}$ values. Also the enhancement proposed by $\mathrm{DTI}_{\text {neigh }}$ did not seem capable of overcoming this intrinsic limitation of DTI. DTI $I_{\text {neigh }}$ can extrapolate additional information about the underlying fiber structure in a voxel (e.g. infer a crossing) by looking at adjacent voxels, and the best results are obtained when in the neighborhood there are single fiber profiles pointing at that voxel. Hence, the compact configuration of bundles present in the SF dataset might have limited its performance.

Despite the wide variety of sampling schemes employed, all remaining approaches were characterized by moderate results in the estimation of the number of fiber populations, with $S R$ always between $50 \%$ and $70 \%$, but with very good performances in terms of angular accuracy, with $\bar{\theta}$ always below $10^{\circ}$. At the extreme side of the sampling panorama, DSI-like methods showed reconstructions with the topmost quality (highest $S R$ and lowest $\bar{\theta}$ values) and remarkably stable (fewer outliers, i.e. red dots). These approaches are 

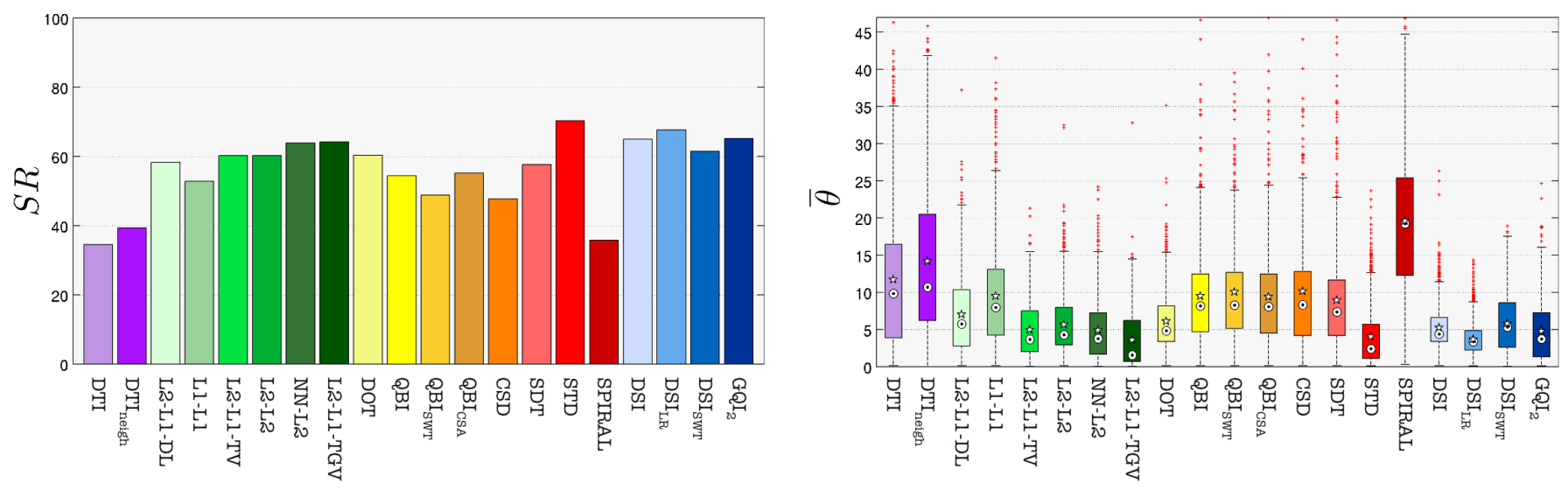

Fig. 2. Overall performances on the SF dataset. On the left panel, the success rate $S R$ obtained by each algorithm in all voxels of the SF phantom is reported using the same color conventions as in Table I. On the right panel, the distributions of the angular accuracy $\bar{\theta}$ are presented as box-and-whisker diagrams. In each box, the edges of the box represent the $25^{\text {th }}$ and $75^{\text {th }}$ percentiles, while the mean and the median are reported as "star" and "circle" marks, respectively. The whiskers extend to the smallest and largest observations in the data, with the values considered as outliers individually plotted as red dots. Results refer to the simulations with $\mathrm{SNR}=30$.

characterized by a very dense acquisition of the signal (257 samples) and the price to pay is a very long scan time which is normally not suitable in a clinical environment.

A sensible reduction in the number of samples (64 to 82) is possible with HARDI-like techniques. However, the reconstructions looked very unstable and presented many outliers which inevitably deteriorated the average performance. The algorithm SPIRAL showed particularly poor scores, but these are compatible with the previous remark on the number of estimated orientations fixed a priori. As an exception the STD method, which is based on symmetric tensor decomposition, obtained very interesting performances rivaling with those of $\mathrm{DSI}_{\mathrm{LR}}$, but using only a quarter of the samples. In particular, these high performances seem to be driven especially by its superior ability to correctly identify the right number of fiber populations in each voxel, as reflected by the highest success rate among all methods $(S R \approx 70 \%$ ).

SPARSE-like methods not only made it possible to further reduce the number of samples (15 to 64) but, interestingly, these algorithms exposed reconstructions with quality comparable to those of DSI-like approaches. In this case, however, despite the fact that $S R$ values were in line with all other methods, the orientation of the fiber populations turned out to be as accurate as more demanding and complex techniques. Considering that SPARSE-like methods only require between $6 \%$ and $25 \%$ the number of samples needed by DSI-like ones, these results can be considered a remarkable achievement which opens the way to attain the quality of HARDI/DSI acquisitions at the price of DTI.

For sake of completeness, during the contest we had also compared the reconstructions with respect to the accuracy in the estimation of the $O D F$. However, not all the algorithms directly recover an ODF; Fig. 5 shows some representative diffusion profiles estimated by each reconstruction method. Consequently, we decided not to report the results of this analysis in the manuscript as this evaluation did not add any informative value to the comparison. The interested reader can find more information on the challenge website.

\section{B. Number of fiber populations}

Fig. 3 presents the detailed performance of each method with respect to the estimation of the number of fiber populations in a voxel. The average values of the three metrics $S R, n^{+}$and $n^{-}$are reported as a function of the crossing angle, where the angle between the two fiber populations was increased from $0^{\circ}$ to $90^{\circ}$. Results refer to the numerical simulations performed on the IV dataset with a $S N R=30$.

At a first glance, it is apparent that the main source of inaccuracy for all reconstruction methods is mainly due to under-estimation other than over-estimation. Over-estimated directions are rather mild, with $n^{+}$always below 0.20 0.25 , and happen mostly at high rather than low crossing angles. On average, fiber compartments started to be underestimated at $60^{\circ}$ and a plateau was reached at about $30^{\circ}$. Above and below these thresholds all algorithms performed essentially the same. Surely DTI always under-estimates one of the two fiber populations and $n^{-}$is always 1 . In the mid-range of crossing angles, DSI-like approaches behaved very well $(52 \%<S R<80 \%)$, while the algorithms in the HARDI-like class, especially DOT and all those based on Q-Ball Imaging, appeared to suffer more $(S R \approx 25-30 \%)$ and the effects of under-estimation started at higher crossing angles. Concerning spherical deconvolution methods, we can see that results are quite heterogeneous. CSD was found to be the method suffering the most from under-estimation in the HARDI-like class $(S R \approx 20 \%)$, while SDT appeared moderately more robust ( $S R \approx 45 \%$ ). STD, on the contrary, performed very well and exhibited the best success rate among all methods $(S R \approx 88 \%$ ), which is linked to a remarkably low rate of under-estimation $\left(n^{-} \approx 0.05\right)$. This method implemented an iterative heuristic to accurately assess the number of compartments in each voxel: a configuration with one fiber population was first reconstructed, then with two etc, and finally the configuration best fitting the data was retained. This procedure, in combination with spherical deconvolution, appeared particularly effective and was able to reliably resolve two fiber populations down to a crossing angle of $30^{\circ}-35^{\circ}$, 

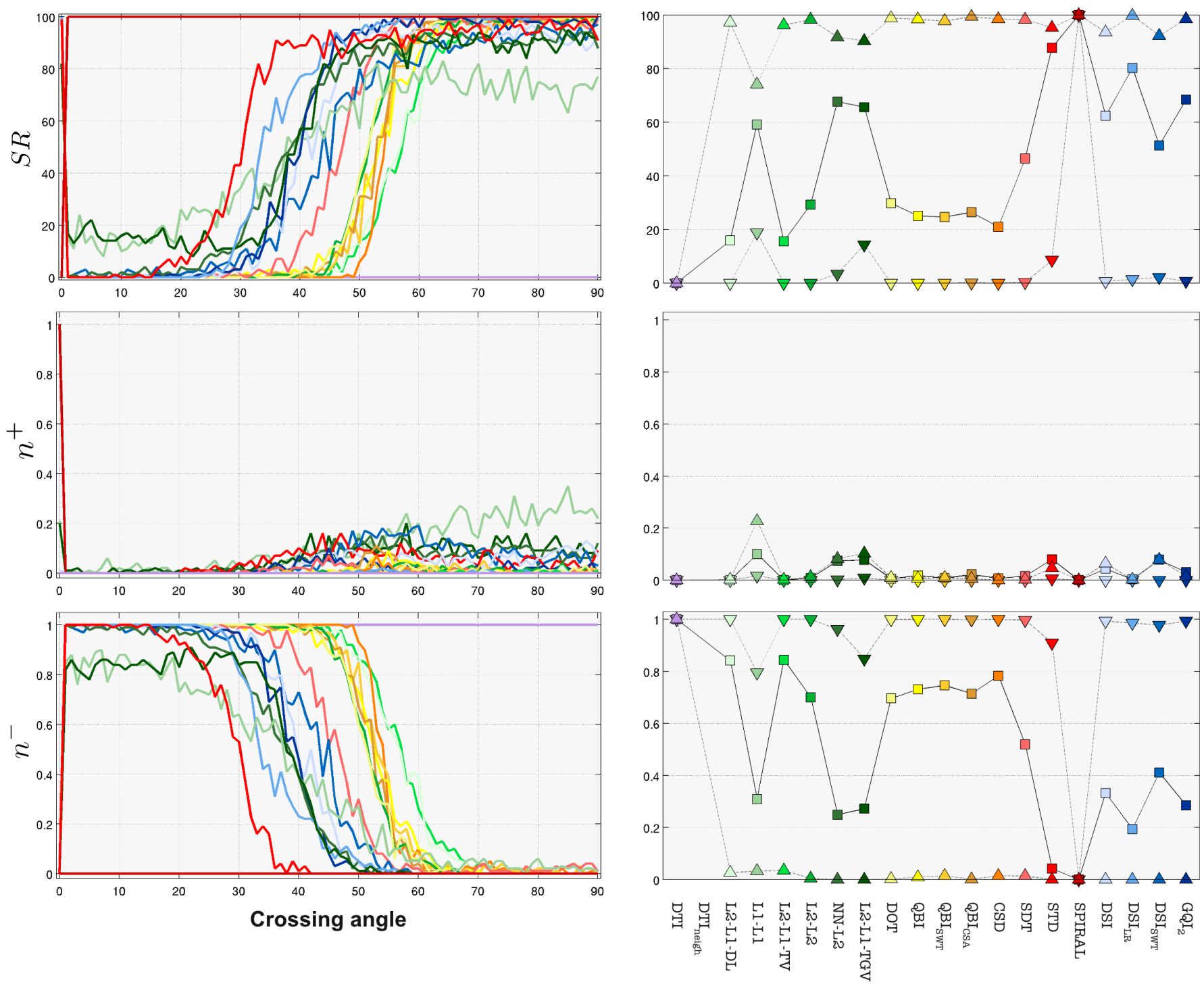

Fig. 3. Estimation of the number of fiber populations as a function of the crossing angle, without using a tolerance cone. The metrics $S R, n^{+}$and $n^{-}$ are reported as the average value across all fiber configurations in the IV dataset crossing at a given angle, in the range $0^{\circ}-90^{\circ}$ (leftmost plots). To help the reader in highlighting differences and similarities among the algorithms, rightmost plots summarize the performances in three separate ranges of crossing angles: $0^{\circ}-30^{\circ}$ (downward triangles), $31^{\circ}-60^{\circ}$ (squares) and $61^{\circ}-90^{\circ}$ (upward triangles). Results correspond to $\mathrm{SNR}=30$.

with $S R \approx 90 \%$ at $35^{\circ}$.

In light of the significant under-sampling rates they permit, SPARSE-like methods exhibited impressive performances. Overall, all algorithms performed at least as good as HARDI-like approaches, with the scores of NN-L2, L1-L1 and L2-L1-TGV competing even with those of DSI $(S R \approx$ 65-70\%). Remarkably, L1-L1 and L2-L1-TGV showed the lowest number of under-estimated compartments at low crossing angles, even better than DSI-like methods. L1-L1 appeared to have some difficulties at large crossing angles, where it showed a higher number of over-estimated compartments. This behavior is most probably linked to the $\ell_{1}$ norm used as goodness measure for the data term in the optimization procedure. Overall, though, these methods performed as good as DSI-like ones but, and it is important to emphasize this aspect, they required only $9 \%-25 \%$ of the samples.

Interesting observations can be made by repeating the pre- vious analysis and considering a tolerance cone around each ground-truth fiber population, i.e. $S R_{\angle}, n_{\perp}^{+}$and $n_{\angle}^{-}$metrics. Comparing Fig. 4 with Fig. 3 we confirm what previously noted, in that differences among algorithms are restricted only to the mid-range of crossing angles, see $S R_{\angle}$ plot in $30^{\circ}-60^{\circ}$, and that the main source of inaccuracy is caused by under-estimation $\left(n_{\bar{L}}^{-}\right.$plot). However, in this case both $n_{\angle}^{+}$and $n_{\perp}^{-}$scores reach much higher values than before, respectively up to 0.7 and 1.7 . As we spotted that the tendency is to under-estimate and $n^{-}$is at worst 1 , this means that the single estimated direction must be outside both tolerance cones around the true fiber orientations: this behavior is in fact captured both as missing either of them (increased $n_{<}^{-}$ values) and also as recovering a spurious one (increased $n_{L}^{+}$ values). DTI is the archetype of this situation: it obtained in fact the highest $n_{<}^{+}$score, almost 1 for crossing angles above $50^{\circ}$, which is clearly counter-intuitive for the nature of the 

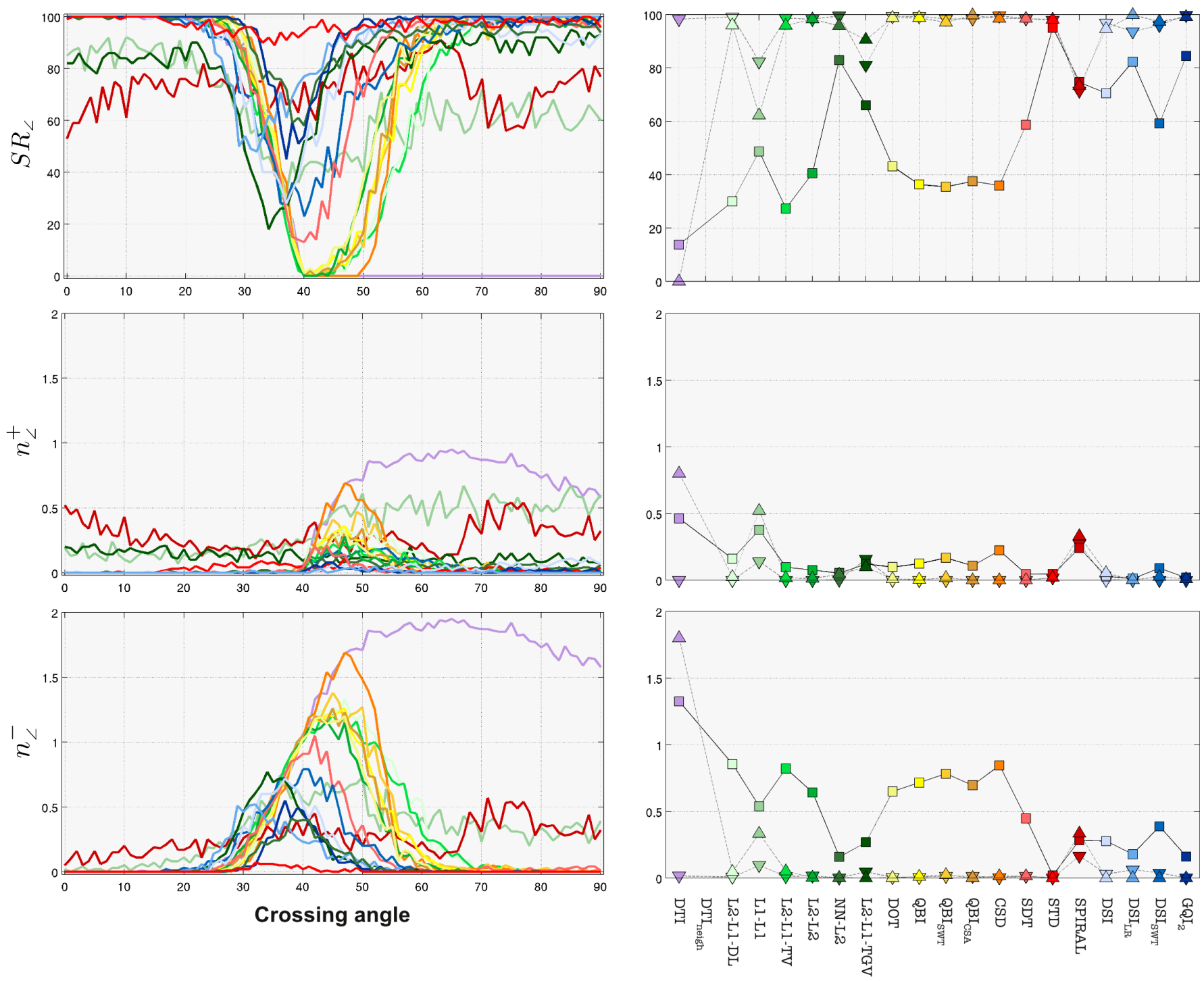

Fig. 4. Estimation of the number of fiber populations as a function of the crossing angle, using a tolerance cone. The metrics $S R_{\angle}$, $n_{\angle}^{+}$and $n_{\angle}^{-}$are reported as the average value across all fiber configurations in the IV dataset crossing at a given angle, in the range $0^{\circ}-90^{\circ}$ (leftmost plots). To help the reader in highlighting differences and similarities among the algorithms, rightmost plots summarize the performances in three separate ranges of crossing angles: $0^{\circ}-30^{\circ}$ (downward triangles), $31^{\circ}-60^{\circ}$ (squares) and $61^{\circ}-90^{\circ}$ (upward triangles). Results correspond to $\mathrm{SNR}=30$.

technique, but in accordance with the estimation of one single direction which lies somewhere between the two real ones. As before, the method STD achieved superb performances, with $S R_{\angle}$ always above $90 \%$ for all crossing angles. Also the performances of SPIRAL seemed almost independent from the crossing angle but, in comparison, its scores were rather modest $\left(S R_{\angle} \approx 70 \%\right)$, once more probably caused by the hard-constraint set internally. Curiously, L1-L1 and L2-L1-TGV turned out to have the lowest $S R_{\angle}$ scores in the crossing range $0^{\circ}-30^{\circ}$ even though, in the same range, they showed the highest $S R$, which was mainly driven by a reduced rate of under-estimation, $n^{-}$, and no over-estimation, $n^{+}$. However, both algorithms showed also a higher $n_{\angle}^{+}$rate, $\approx 0.2$, which is an indication that one of the two directions estimated is not close to the corresponding true one and is considered spurious.

We conclude this section by noting two drawbacks of this latter set of metrics, i.e. $S R_{\angle}, n_{\angle}^{+}$and $n_{\angle}^{-}$. First, for configurations with crossing angle less than $40^{\circ}$ these indices become ambiguous, as in this case the tolerance cones of the two fiber populations unavoidably overlap. However, above $40^{\circ}$ they essentially describe the same trends. Second, they are very dependent on the used threshold, $20^{\circ}$ in this study. For these reasons and for compactness of the exposition, we decided to report in the remaining only the measures without tolerance cone, i.e. $S R, n^{+}$and $n^{-}$, as they appeared to us more intuitive and sufficiently informative for the scope of this work. All evaluations are available online.

\section{Angular accuracy}

Fig. 6 compares all reconstruction methods with respect to the accuracy in the orientation of the estimated fiber populations, $\bar{\theta}$, as a function of the crossing angle. Results refer to the simulations performed with a $\mathrm{SNR}=30$.

DTI is easily recognizable. The fact that $\bar{\theta}$ is approximately 

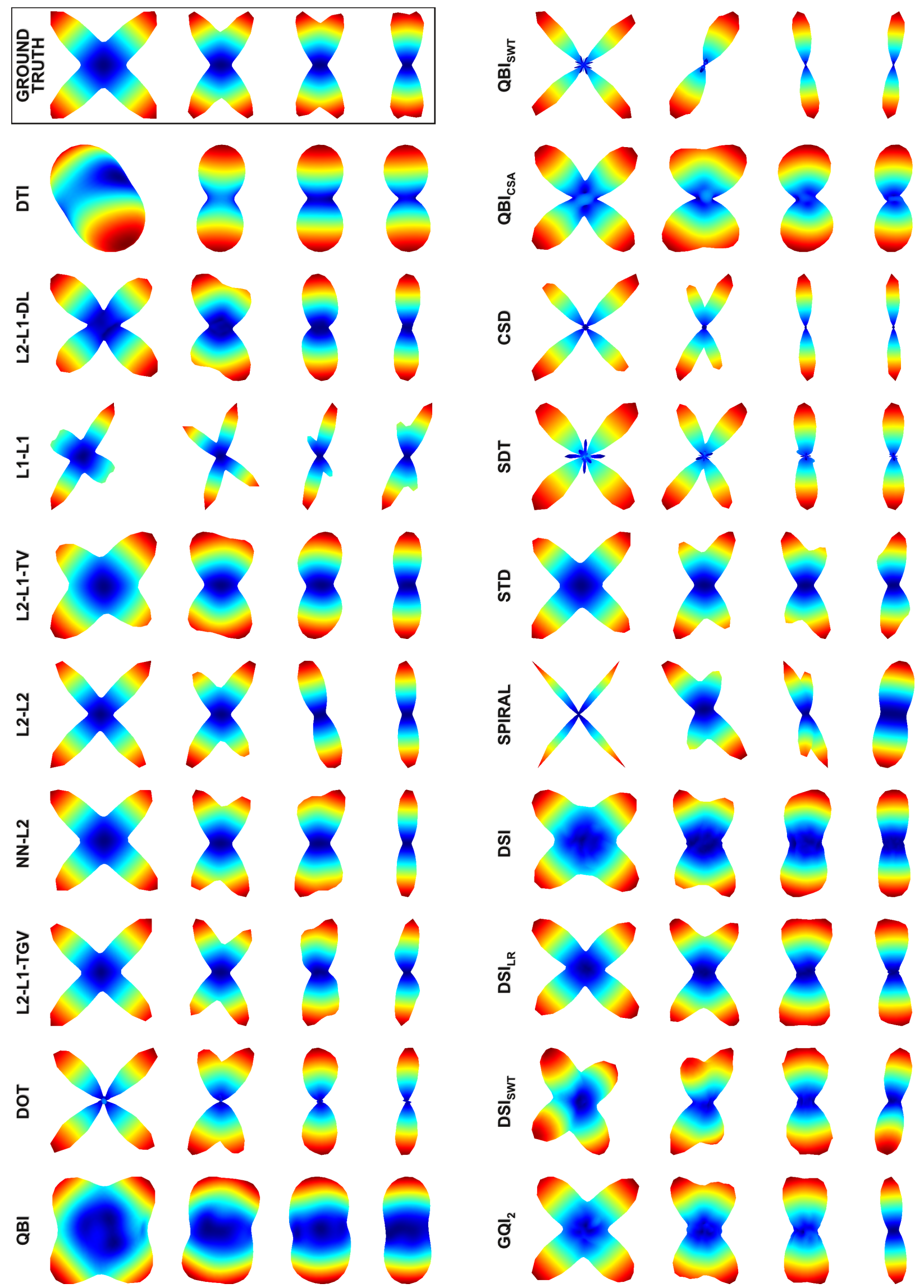

Fig. 5. Estimated diffusion features. One representative diffusion profile as reconstructed by each algorithm (e.g. ODF or FOD) is shown for four different crossing configurations $\left(90^{\circ}, 60^{\circ}, 45^{\circ}, 30^{\circ}\right)$. Results correspond to the reconstructions on the IV dataset with a SNR=30. As these data were not available for method DTI $\mathrm{I}_{\text {neigh }}$, the corresponding profiles are not shown. 

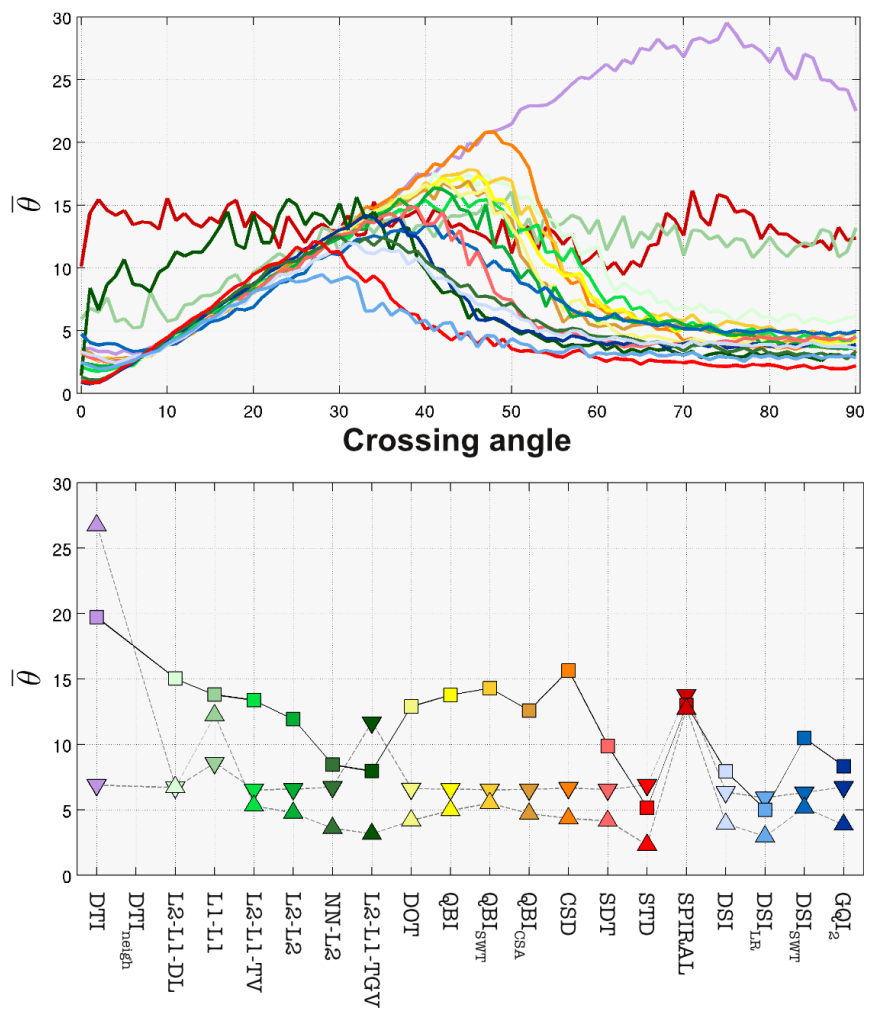

Fig. 6. Accuracy in the orientation of the estimated fiber compartments as a function of the crossing angle. The metric $\bar{\theta}$ is reported (in degrees) as the average value across all fiber configurations in the IV dataset crossing at a given angle, in the range $0^{\circ}-90^{\circ}$ (top plot). As for Fig. 3 and Fig. 4 , the average performance is also reported in three separate ranges of crossing angles (bottom plot): $0^{\circ}-30^{\circ}$ (downward triangles), $31^{\circ}-60^{\circ}$ (squares) and $61^{\circ}-90^{\circ}$ (upward triangles). Results correspond to $\mathrm{SNR}=30$.

half of the angle separating the two ground-truth directions, together with the observations in the previous paragraph, is compatible with the reconstruction of a single orientation lying in the middle of them and, in practice, it identifies a boundary under which the technique is unable to resolve multiple directions. This limit was reached by all the methods as the crossing angle between the two fiber populations decreased: it happened at $48^{\circ}$ for the CSD algorithm while the limit was $25^{\circ}$ in the case of STD and $\mathrm{DSI}_{\mathrm{LR}}$.

Despite quite heterogeneous results obtained concerning the estimation of the number of fiber populations, these variations translated only to fairly small differences between methods with regard to the orientation of the estimated directions. All tested algorithms exposed very high angular accuracy both at low and high crossing angles $\left(\bar{\theta} \approx 3^{\circ}-7^{\circ}\right)$, while in the mid-range the performances quickly deteriorated. The largest difference between algorithms was observed at a crossing angle of $48^{\circ}$, where $\bar{\theta}$ varied between $4^{\circ}$ in case of both STD and DSI $I_{\mathrm{LR}}$ and $21^{\circ}$ for CSD. The observed tendency of the L1-L1 algorithm to over-estimate compartments at high crossing angles had quite a severe impact on the angular accuracy of the corresponding reconstructions $\left(\bar{\theta} \approx 13^{\circ}\right)$, three times higher than the average performance of all the other algorithms $\left(\bar{\theta} \approx 4^{\circ}\right)$. At low crossing angles, on the other hand, the $\bar{\theta}$ values for both L1-L1 and L2-L1-TGV were higher than the average angular error of all other methods, even thought they previously had shown the best under-estimation behavior $\left(n^{-} \approx 0.8\right)$ and over-estimation was almost absent $\left(n^{+} \approx 0\right)$. Inspecting the corresponding reconstructions we could observe that both algorithms normally recovered one fiber lying halfway the two true peaks like all others algorithms but, additionally, they reconstructed very often a spurious fiber direction (captured in $n_{\angle}^{+}$plots). This extra compartment explains both the reduced under-estimation rate exposed by these algorithms and, as it was normally orientated randomly, the drop in the average angular accuracy of the reconstructions.

Special reference needs to be made about the algorithm SPIRAL, as the angular error appeared to be in the average range but almost independent from the crossing angle. The reasons for this trend are not clear, but it might be a side effect of the procedure used to recover their orientations. The authors used in fact the 82 sampling directions both to represent the signal and estimate the fiber directions, and thus the granularity of this grid (the points are separated by about $20^{\circ}$ ) might have hampered the performances of their method.

\section{Robustness to noise}

Fig. 7 compares the algorithms with respect to the sensitivity to noise of the corresponding reconstructions, reported in the plots as the variation of the three metrics $\bar{\theta}, n^{+}$and $n^{-}$as the acquisition SNR decreases. The performances obtained at a $\mathrm{SNR}=30$ are taken as reference and the variation of the scores is reported for $\mathrm{SNR}=20$ and $\mathrm{SNR}=10$, which resemble more closely the typical SNR in real dMRI data. Positive values mean a degradation of the performances.

As expected, the angular resolution of the reconstructions gradually deteriorated as the noise increased, with no clear differences among the algorithms. Yet, methods with higher number of samples seemed to be in general more stable. DTI and SPIRAL appeared the most robust to noise $\left(\Delta_{\bar{\theta}}<4^{\circ}\right)$ while DSI the most susceptible, especially at $\mathrm{SNR}=10$ where it degraded abruptly showing a $\Delta_{\bar{\theta}} \approx 21^{\circ}$. However, it is worth noting how the enhancements implemented in $\mathrm{DSI}_{\mathrm{LR}}$ and $\mathrm{DSI}_{\mathrm{SWT}}$ allowed a significant boost in the robustness to noise in methods based on Cartesian signal sampling, turning standard DSI from the most susceptible to noise method to the most robust. Also CSD showed very stable performances, appearing to suffer only at high crossing angles, despite no significant worsening in under- and over-estimation rates.

In general, all methods showed the highest susceptibility to noise and, correspondingly, the major drop in the performances at medium-high crossing angles (boxes and upward triangles), with drops in the angular accuracy $\Delta_{\bar{\theta}}$ of the reconstructions up to $8^{\circ}-11^{\circ}$. In particular, this degradation seemed driven mostly by over-estimation, as easily visible in the $\Delta_{n^{+}}$plot. This observation is in accordance with the tendency of all methods, previously observed in Fig. 3, to recover spurious compartments at high, more than at low, crossing angles. On the other hand, high noise levels induced an opposite behavior in algorithms using highly under-sampled acquisition schemes, like L2-L1-DL, L1-L1 and L2-L1-TV, with less than 30 samples. In fact, these methods did not show an increased rate of overestimated compartments but, on the contrary, an higher number 

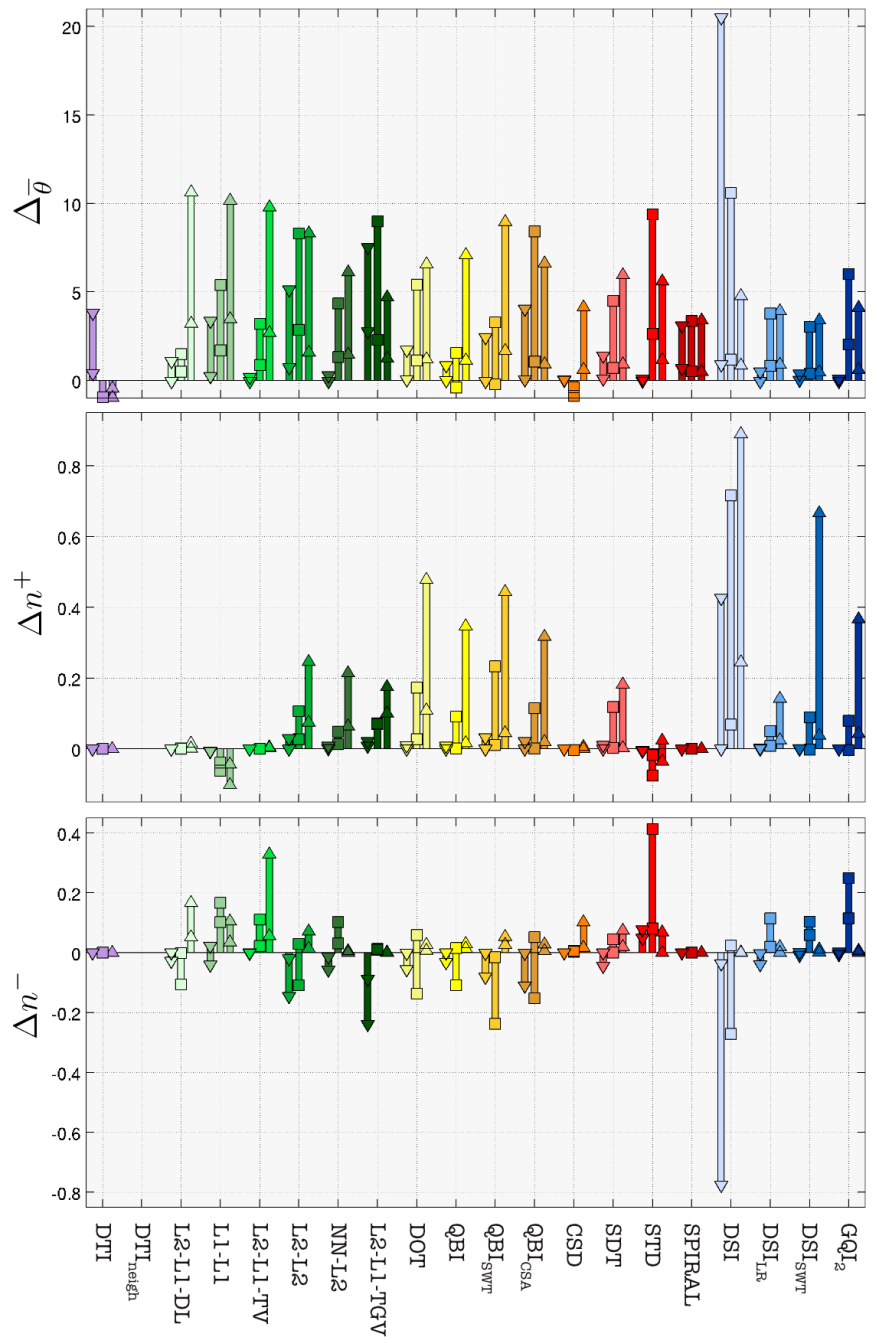

Fig. 7. Overall performances as a function of the noise level. For each method, the variations of the estimated number, $n^{+}$and $n^{-}$, and orientation, $\bar{\theta}$, of the fiber populations are reported as the noise level changes. The first mark of every bar corresponds to the difference between the scores at $\mathrm{SNR}=30$ and those at $\mathrm{SNR}=20$, while the second mark between $\mathrm{SNR}=30$ and $\mathrm{SNR}=10$. Results refer to the simulations performed on the IV datasets and, as usual, are reported separately for three crossing angle ranges: $0^{\circ}-30^{\circ}$ (downward triangles), $31^{\circ}-60^{\circ}$ (squares) and $61^{\circ}-90^{\circ}$ (upward triangles).

of missed fibers. The method STD showed the same tendency to under-estimate more with higher noise levels and it resulted the most prone to under-estimation at $\mathrm{SNR}=10$. Interestingly, the ability of STD to under-estimate less than all other algorithms in the medium range of crossing angles was the reason behind its exquisite performances ( $n^{-}$plot in Fig. 3). STD performed very well with low noise, but unfortunately this robustness to missing fibers rapidly degraded as the noise increased. Curiously, the $\Delta_{n^{-}}$plot in Fig. 7 shows that most of the algorithms got some benefit $\left(\Delta_{n^{-}}<0\right)$ from higher noise levels at low-medium crossing angles (boxes and downward triangles), with DSI being the most "beneficial". However, this strange behavior is a side-effect of over-estimation. In fact, at $\mathrm{SNR}=30$, all methods were shown to suffer heavily from under-estimation for crossing angles below $60^{\circ}$ (see $n^{-}$plot in Fig. 3). Higher noise levels caused an increased rate of spurious fiber compartments recovered, which not only were clearly captured by the positive values in the $\Delta_{n^{+}}$plot, but also by negative $\Delta_{n^{-}}$values.

The observed tendency to recover spurious compartments as the noise increases can be explained, in part, by the fact that all the algorithms considered in this work are based on the assumption that the distribution of the noise follows a Gaussian distribution with zero mean. However, the noise corrupting the data follows a Rician distribution and the deviation from Gaussianity is particularly evident at low SNR values [61]. Rician noise produces, on average, a net increase in the signal which is different along every gradient direction. As a result, reconstructions assuming zero-mean Gaussian noise tend to produce small spurious fibers to explain this discrepancy.

As last remark, the results obtained in our study are strictly valid only for single-channel or SENSE multi-channel acquisitions [70] and they should not be extrapolated to other parallel techniques such as GRAPPA [71], where the noise follows a noncentral- $\chi$ distribution. Please note however that nearly all reconstruction methods proposed to date were tested only on Rician-corrupted data, i.e. using the same assumption, and thus our results are consistent with previous literature.
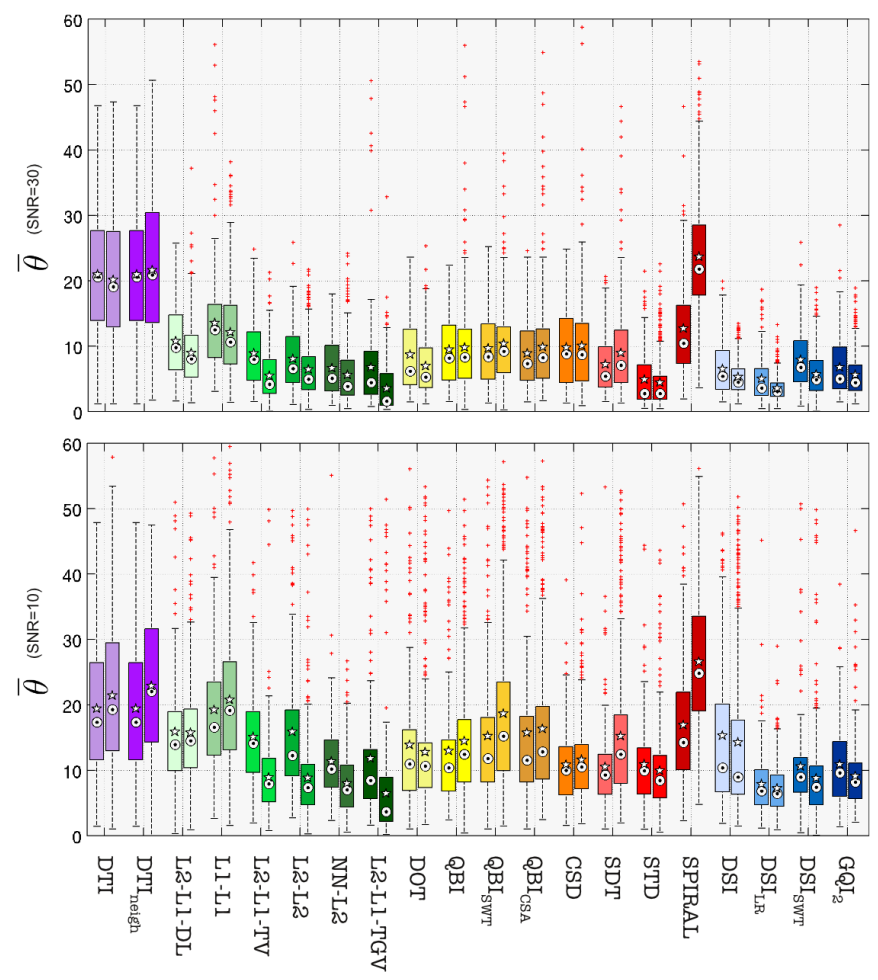

Fig. 8. Effects of using information from neighboring voxels on the quality of reconstructions. For each method, the distribution of the average angular errors $\bar{\theta}$ obtained on the IV phantom (left bar) are compared with those corresponding to SF (right bar). Results are reported for two noise levels: $\mathrm{SNR}=30$ (top) and SNR=10 (bottom).

\section{E. Spatial neighborhood}

Fig. 8 investigates the effect of using additional information from neighboring voxels on the quality of reconstructions. For each method, we compared the distributions of the average angular errors $\bar{\theta}$ obtained on the IV (left bar) and SF (right bar) phantoms. To be sensitive to this effect only, we considered a 
subset of voxels with two fiber populations from both datasets and we matched the distributions of the crossing angles. We compared the results corresponding to low and high noise levels, respectively with SNR=30 (top) and SNR=10 (bottom).

The algorithms reported in Table I to exploit additional information from neighboring voxels in some form, either performing spatial smoothing on the DWI images or employing a regularization scheme, are clearly identifiable. For these methods, in fact, the angular accuracy of the reconstructions clearly benefitted (smaller $\bar{\theta}$ errors) from the spatial coherence among voxels present in the SF phantom (right bar in each method's results) if compared to the results obtained on the IV dataset (left bar), which indeed consists of un-correlated voxels. These de-noising approaches appeared definitely more effective on data corrupted with higher noise levels, as apparent in the plot corresponding to $\mathrm{SNR}=10$, where improvements in the angular accuracy up to $7^{\circ}-8^{\circ}$ can be appreciated. On the other hand, with high quality data the regularization-based approaches appeared more effective than those using spatial smoothing. In this case, in fact, these latter did not seem to give any benefit (see performances of L2-L2 and NN-L2 at SNR=30) while methods using spatial regularization schemes, i.e. L2-L1-TV and L2-L1-TGV, still provided a $3^{\circ}-4^{\circ}$ improvement in the average angular accuracy.

Surprisingly, even though we did not expect any significant variation for those methods not exploiting any spatial coherence in the data, a curious behavior can still be observed in the figure. In fact, some of the approaches in the HARDI-like class showed a performance reduction when recovering the intravoxels structure in the SF dataset, which is sort of counterintuitive. One possible speculation for this odd behavior can be attributable to different settings for the parameters used in the two datasets. As the structure of the SF dataset did include voxels with more than two crossing fibers (even though these configurations were excluded from the evaluation), more tolerant thresholds have been probably used in this case.

\section{F. Signal-to-noise ratio and voxel-size}

Because of the assumption on $S_{0}$ we made in section II-B, in our experiments we have implicitly considered a constant echo-time (TE) for all acquisition protocols, hence neglecting the additional dependence of the signal on the damping factor $\exp (-\mathrm{TE} / \mathrm{T} 2)$, where $\mathrm{T} 2$ is the spin-spin relaxation time of the tissue. However, in clinical systems, increasing the b-value inevitably implies a longer TE which, in turn, negatively impacts the effective SNR of the acquisitions. To obtain images with adequate SNR, thus, clinical protocols typically adapt the spatial resolution and use a rather large voxel-size for high bvalue sequences such as DSI, e.g. $2.5 \times 2.5 \times 2.5 \mathrm{~mm}^{3}$, whereas DTI is normally acquired with $1.8 \times 1.8 \times 1.8 \mathrm{~mm}^{3}$ resolution.

Fig. 9 shows a further comparison between the reconstructions performed on the original data and those repeated after adjusting the noise level to account for the specific TE of each sequence. For compactness the results are reported only for the most popular techniques, such as DTI, CSD and DSI. The new adjusted SNRs reported in Table II have been computed taking the protocol with the longest TE as reference
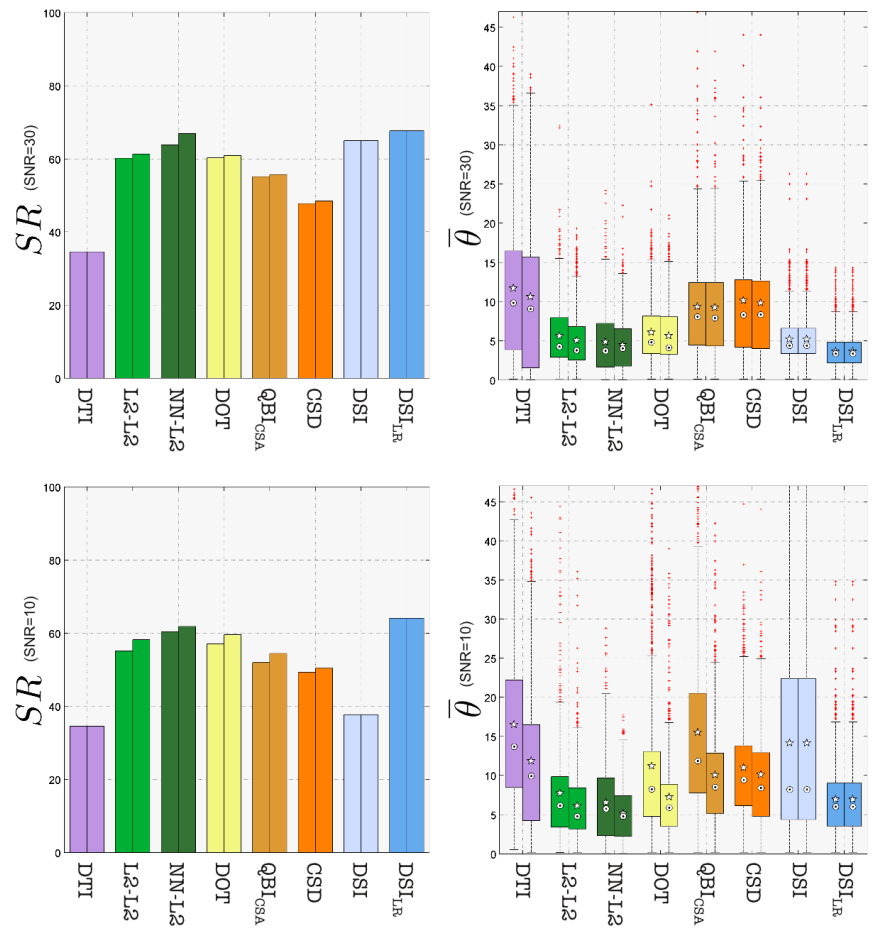

Fig. 9. Comparison between "uncorrected" and "adjusted" SNRs. The overall $S R$ and $\bar{\theta}$ performances on the SF dataset are reported, for a subset of the algorithms, before (left bar of each method) and after (right bar) correction of the effective signal-to-noise ratio. Results are shown for two uncorrected noise levels: $\mathrm{SNR}=30$ (top) and $\mathrm{SNR}=10$ (bottom).

and assuming the same spatial resolution, yielding reasonable values commonly observed in real datasets. Realistic TE values have been extracted from a clinical 3T scanner with maximum gradient strength of $40 \mathrm{mT} / \mathrm{m}$, and the T2 in the white matter was assumed equal to $80 \mathrm{~ms}$ as found in [72].

TABLE II

ADJUSTED SNRS BY CONSIDERING EACH SEQUENCE ECHO-TIME (TE).

\begin{tabular}{|l|ccccccccc|}
\hline b-value & 750 & 1000 & 1200 & 1500 & 2000 & 2500 & 3000 & 3333 & 8000 \\
TE & 80 & 84 & 87 & 91 & 98 & 107 & 114 & 118 & 162 \\
\hline \multirow{3}{*}{ SNR } & 83.6 & 79.5 & 76.6 & 72.9 & 66.8 & 59.7 & 54.7 & 52.0 & 30 \\
& 55.7 & 53.0 & 51.1 & 48.6 & 44.5 & 39.8 & 36.4 & 34.7 & 20 \\
& 27.9 & 26.5 & 25.5 & 24.3 & 22.3 & 19.9 & 18.2 & 17.3 & 10 \\
\hline
\end{tabular}

The performances of DSI and DSI $I_{\mathrm{LR}}$ are clearly constant as their SNR remained fixed. Also the success rate of DTI did not change, but this is due to the intrinsic limitation of the model. As previously reported in Fig. 7, all methods can provide better reconstructions when working on images with higher quality, but they are characterized by different levels of robustness to the noise. At a noise level typically observed in real acquisitions (upper plots), in fact, no significant improvement can be noted for any of the methods when increasing the effective SNR to account for shorter TE, both in terms of $S R$ and $\bar{\theta}$. With very low image quality (bottom plots) the methods with shorter TE ameliorate the reconstructions, even though clearly the same trend of performances is preserved across methods. Hence, for the scope of this study, the effect of each sequence echo-time on the effective SNR of the data did not seem to introduce significant bias in the results. 


\section{G. Dependence on the signal model}

During the contest some concerns arose with respect to the Gaussian Mixture Model (1) that was used to simulate the dMRI signal in place of the more precise expression for particles diffusing in a restricted cylindrical geometry given in [58]. Both models have been largely used in the literature for validation of diffusion estimators, as well as the use of physical phantoms. As already pointed out in the introduction, all these models are undoubtedly simplifications of the actual biophysical processes occurring in white matter. In fact, for a more accurate synthetic simulation, it should be taken into account the complex axonal structure, the membrane permeability, the bi-exponential behavior of the signal with respect to the b-value magnitude, the existence of slow and fast pools etc. In the particular case of the contest, however, the main concern was that our choice might have favored the model-based reconstruction algorithms, such as those in the SPARSE-like class, using implicitly or explicitly the same Gaussian assumption.

To investigate any possible influence of this choice on the reconstructions considered in our study, we simulated the dMRI signal attenuation corresponding to a single fiber configuration using both expressions: (i) Gaussian Mixture Model [8] and (ii) Soderman's Model [58]. Fig. 10 compares the signal decay as predicted by the two models, named MultiTensor and Soderman in the plot, as a function of the b-value. For the latter, we used the following parameters as reported in previous studies [20], [34]: $L=5 \mathrm{~mm}, \rho=5 \mu \mathrm{m}$, $D_{0}=2.02 \times 10^{-3} \mathrm{~mm}^{2} / \mathrm{s}$, but we set $\Delta=80 \mathrm{~ms}$ and $\delta=35 \mathrm{~ms}$ to resemble an acquisition with maximum b-value of $4000 \mathrm{~s} / \mathrm{mm}^{2}$ on a clinical 3T scanner with a maximal gradient strength of $40 \mathrm{mT} / \mathrm{m}$ [68]. To simulate the signal with the model (1) and match the diffusion pattern, a single tensor was fitted to the data and the estimated parameters were used for the simulation. The diffusivities along the three main axes were found to be: $\lambda_{1}=2.3 \times 10^{-3} \mathrm{~mm}^{2} / \mathrm{s}$ and $\lambda_{2,3}=0.1 \times 10^{-3} \mathrm{~mm}^{2} / \mathrm{s}$.

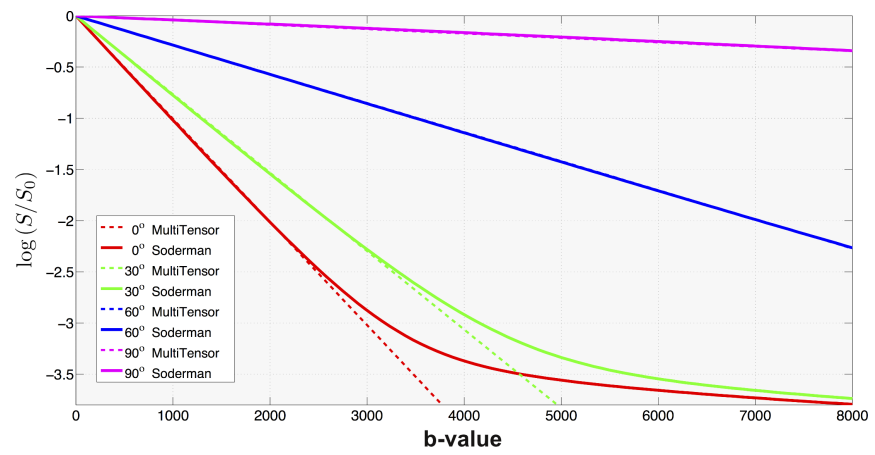

Fig. 10. dMRI signal attenuation of a single fiber configuration simulated using two different models. The decays predicted by the MultiTensor [8] (dashed lines) and the Soderman [58] (solid lines) models are plotted as a function of the b-value. Results are reported at four different angles between the diffusion gradient and the fiber population $\left(0^{\circ}, 30^{\circ}, 60^{\circ}, 90^{\circ}\right)$.

As we can see, the signal decays predicted by the two models, dashed lines for MultiTensor and solid for Soderman, appear almost indistinguishable for b-values up to $3000 \mathrm{~s} / \mathrm{mm}^{2}$, with differences becoming appreciable (note the logarithmic scale) above $4000 \mathrm{~s} / \mathrm{mm}^{2}$, especially when the diffusion gradient is parallel to the orientation of the fiber (red curves). Interestingly, 16 of the 20 algorithms evaluated in this study employed acquisition schemes with a maximum b-value of about $3000 \mathrm{~s} / \mathrm{mm}^{2}$. Furthermore, the remaining 4 methods belonged to the DSI-like class and were all characterized by model-free approaches for the reconstruction of the intravoxel fiber configuration. In light of these arguments, we genuinely believe that for the scope of this study the choice of the model (1) that we used in our numerical simulations for the generation of the diffusion signal did not favor those model-based approaches which made use of the same Gaussian assumption and, for this reason, did not bias the comparison.

\section{H. General observations}

It is always difficult to draw general conclusions from comparative studies without going into the details of every single algorithm evaluated. This is particularly true in this work as the evaluated approaches differ in a great deal of aspects including acquisition scheme, assumptions made in the modeling (i.e. model-free and model-based approaches were included), numerical algorithms employed in the estimation process, descriptors used to characterize the intravoxel structure (i.e. diffusion ODF or FOD), as well as some heuristic parameters such as the threshold used to select the main ODF local maxima and the regularization parameters. For this reason, during the "HARDI reconstruction challenge" we decided not to provide a final ranking based on scores to highlight a winner. This decision was mainly motivated due to the lack of a fair quantitative model to integrate all the different aspects involving each method.

In contrast, in order to help clinicians in the choice of the most adequate technique for a specific application according to their interests and possibilities, we summarize here the main lessons we have learned from this study:

- None of the methods was found to outperform the others in every experimental condition. On the contrary, results suggest that a combination of techniques might be the way to obtain optimal reconstructions.

- Overall, $\mathrm{DSI}_{\mathrm{LR}}$ appeared the most accurate and stable (i.e. fewer outliers), but the long scan time highly restricts its applicability in clinical environments.

- Accurate reconstructions (comparable to those obtained from HARDI/DSI) at the price of DTI acquisitions are becoming possible thanks to SPARSE-like approaches, which in general showed very interesting performances.

- With high quality data the main source of inaccuracy for all the algorithms seemed to be caused by underestimation. On the other hand, for low/medium SNR acquisitions the degradation of the performances was mainly driven by over-estimation.

- Noise removal procedures, either in the form of image denoising or spatial regularization, are highly recommended as results showed they can improve the performances up to $30-40 \%$. We did not observe any substantial difference in the efficacy of the two approaches; nevertheless, spatial regularization appeared to be effective also in low noise regimes, while merely denoising the images did not. 
It is important to remark that the results presented in this work are based on numerical simulations and, as previously mentioned, there is no guarantee that these observations hold true to the same extent also in case of real data.

\section{CONCLUSION}

We have quantitatively evaluated and compared a large selection of algorithms for recovering the local intra-voxel fiber structure from diffusion MRI acquisitions. Considered methods included a mixture of classical and well-established techniques as well as novel approaches recently proposed in the literature, in particular those based on compressed sensing theory. Despite this study has been conducted on synthetic dMRI data, results yet allowed to spot strengths and weaknesses of each approach and to draw interesting considerations common to all the algorithms. We believe that this information might be used both to improve current reconstruction methods and also as a guidance for physicians to identify the most adequate technique for their clinical studies.

\section{ACKNOWLEDGEMENTS}

This work benefited from the precious help of all the contest participants, who provided not only the reconstructions that were evaluated but also assistance and valuable comments during the writing of the present manuscript.

\section{REFERENCES}

[1] C. Beaulieu, "The basis of anisotropic water diffusion in the nervous system - a technical review." NMR Biomed, vol. 15, pp. 435-55, 2002.

[2] T. Conturo, N. Lori, T. Cull, E. Akbudak, A. Snyder, J. Shimony, R. McKinstry, H. Burton, and M. Raichle, "Tracking neuronal fiber pathways in the living human brain.” PNAS, vol. 96, pp. 10422-7, 1999.

[3] S. Mori, B. Crain, V. Chacko, and P. van Zijl, "Three-dimensional tracking of axonal projections in the brain by magnetic resonance imaging." Ann Neurol, vol. 45, pp. 265-9, 1999.

[4] P. Fillard, M. Descoteaux, A. Goh, S. Gouttard, B. Jeurissen, J. Malcolm, A. Ramirez-Manzanares, M. Reisert, K. Sakaie, F. Tensaouti, T. Yo, J.F. Mangin, and C. Poupon, "Quantitative evaluation of 10 tractography algorithms on a realistic diffusion $\mathrm{mr}$ phantom." NeuroImage, vol. 56, pp. 220-34, 2011.

[5] P. Basser, J. Mattiello, and D. LeBihan, "MR diffusion tensor spectroscopy and imaging," Biophys J, vol. 66, pp. 259-67, 1994.

[6] V. Wedeen, P. Hagmann, W.-Y. Tseng, T. Reese, and R. Weisskoff, "Mapping complex tissue architecture with diffusion spectrum magnetic resonance imaging," Magn Reson Med, vol. 54, pp. 1377-86, 2005.

[7] P. Callaghan, A. Coy, D. Macgowan, K. Packer, and F. Zelaya, "Diffraction-like effects in NMR diffusion studies of fluids in porous solids," Nature, vol. 351, pp. 467-9, 1991.

[8] D. Tuch, T. Reese, M. Wiegell, N. Makris, J. Belliveau, and V. Wedeen, "High angular resolution diffusion imaging reveals intravoxel white matter fiber heterogeneity," Magn Reson Med, vol. 48, pp. 577-82, 2002.

[9] T. E. J. Behrens, M. W. Woolrich, M. Jenkinson, H. Johansen-Berg, R. G. Nunes, S. Clare, P. M. Matthews, J. M. Brady, and S. M. Smith, "Characterization and propagation of uncertainty in diffusion-weighted MR imaging," Magn Reson Med, vol. 50, pp. 1077-88, 2003.

[10] B. W. Kreher, J. F. Schneider, I. Mader, E. Martin, J. Hennig, and K. A. Il'yasov, "Multitensor approach for analysis and tracking of complex fiber configurations," Magn Reson Med, vol. 54, pp. 1216-25, 2005.

[11] L. Melie-García, E. J. Canales-Rodríguez, Y. Alemán-Gómez, C. P. Lin Y. Iturria-Medina, and P. A. Valdés-Hernández, "A Bayesian framework to identify principal intravoxel diffusion profiles based on diffusionweighted MR imaging," NeuroImage, vol. 42, pp. 750-70, 2008.

[12] D. C. Alexander, G. J. Barker, and S. R. Arridge, "Detection and modeling of non-Gaussian apparent diffusion coefficient profiles in human brain data," Magn Reson Med, vol. 48, pp. 331-40, 2002.
[13] T. Schultz and H. P. Seidel, "Estimating crossing fibers: a tensor decomposition approach," IEEE Trans Vis Comput Graph, vol. 14, pp. 1635-42, 2008.

[14] A. Barmpoutis, M. S. Hwang, D. Howland, J. R. Forder, and B. C. Vemuri, "Regularized positive-definite fourth order tensor field estimation from DW-MRI," NeuroImage, vol. 45, pp. 153-62, 2009.

[15] D. Tuch, "Q-ball imaging," Magn Reson Med, vol. 52, pp. 1358-72, 2004.

[16] A. Tristán-Vega, C. F. Westin, and S. Aja-Fernández, "Estimation of fiber orientation probability density functions in high angular resolution diffusion imaging," NeuroImage, vol. 47, pp. 638-50, 2009.

[17] I. Aganj, C. Lenglet, G. Sapiro, E. Yacoub, K. Ugurbil, and N. Harel, "Reconstruction of the orientation distribution function in single- and multiple-shell Q-ball imaging within constant solid angle," Magn Reson Med, vol. 64, pp. 554-66, 2010.

[18] K. Jansons and D. Alexander, "Persistent angular structure: new insights from diffusion magnetic resonance imaging data," Inverse Problems, vol. 19, pp. 1031-46, 2003.

[19] Y. Assaf and P. Basser, "Composite hindered and restricted model of diffusion (CHARMED) MR imaging of the human brain," NeuroImage, vol. 27, pp. 48-58, 2005.

[20] E. Özarslan, T. Shepherd, B. Vemuri, S. Blackband, and T. Mareci, "Resolution of complex tissue microarchitecture using the diffusion orientation transform (DOT)," NeuroImage, vol. 31, pp. 1086-103, 2006.

[21] E. Canales-Rodríguez, C.-P. Lin, Y. Iturria-Medina, C.-H. Yeh, K.-H. Cho, and L. Melie-García, "Diffusion orientation transform revisited," NeuroImage, vol. 49, pp. 1326-39, 2009.

[22] Y. Wu and A. Alexander, "Hybrid diffusion imaging," NeuroImage, vol. 36, pp. 617-29, 2007.

[23] F.-C. Yeh, V. Wedeen, and W.-Y. Tseng, "Generalized q-sampling imaging," IEEE Trans Med Imaging, vol. 29, pp. 1626-35, 2010.

[24] A. Anderson and Z. Ding, "Sub-voxel measurement of fiber orientation using high angular resolution diffusion tensor imaging," in Proc. 10th Annual Meeting of the ISMRM, 2002, p. 440.

[25] J. D. Tournier, F. Calamante, D. G. Gadian, and A. Connelly, "Direct estimation of the fiber orientation density function from diffusionweighted MRI data using spherical deconvolution," NeuroImage, vol. 23, pp. 1176-85, 2004.

[26] D. C. Alexander, "Maximum entropy spherical deconvolution for diffusion MRI," in Inf Process Med Imaging, vol. 19, 2005, pp. 76-87.

[27] J. D. Tournier, F. Calamante, and A. Connelly, "Robust determination of the fibre orientation distribution in diffusion MRI: non-negativity constrained super-resolved spherical deconvolution," NeuroImage, vol. 35, pp. 1459-72, 2007.

[28] F. Dell'Acqua, G. Rizzo, P. Scifo, R. A. Clarke, G. Scotti, and F. Fazio, "A model-based deconvolution approach to solve fiber crossing in diffusion-weighted $\mathrm{mr}$ imaging," IEEE Trans Biomed Eng, vol. 54, pp. 462-72, 2007.

[29] M. Descoteaux, R. Deriche, T. Knosche, and A. Anwander, "Deterministic and Probabilistic Tractography Based on Complex Fibre Orientation Distributions," IEEE Trans Med Imaging, vol. 28, pp. 269-86, 2009.

[30] E. Panagiotaki, T. Schneider, B. Siow, M. G. Hall, M. F. Lythgoe, and D. C. Alexander, "Compartment models of the diffusion MR signal in brain white matter: a taxonomy and comparison," NeuroImage, vol. 59, pp. 2241-54, 2012.

[31] D. Donoho, “Compressed sensing," IEEE Trans Inf Theory, vol. 52, pp. 1289-306, 2006.

[32] E. Candès, J. Romberg, and T. Tao, "Robust uncertainty principles: exact signal reconstruction from highly incomplete frequency information," IEEE Trans Inf Theory, vol. 52, pp. 489-509, 2006.

[33] A. Ramirez-Manzanares, M. Rivera, B. C. Vemuri, P. Carney, and T. Mareci, "Diffusion basis functions decomposition for estimating white matter intravoxel fiber geometry," IEEE Trans Med Imaging, vol. 26, pp. 1091-102, 2007.

[34] B. Jian and B. C. Vemuri, "A unified computational framework for deconvolution to reconstruct multiple fibers from diffusion weighted MRI," IEEE Trans Med Imaging, vol. 26, pp. 1464-71, 2007.

[35] A. Tristán-Vega and C.-F. Westin, "Probabilistic ODF estimation from reduced HARDI data with sparse regularization," in Proc. Med. Image. Comput. Comput. Assist. Interv. (MICCAI), 2011, pp. 182-90.

[36] Y. Rathi, O. Michailovich, K. Setsompop, S. Bouix, M. E. Shenton, and C.-F. Westin, "Sparse multi-shell diffusion imaging," in Proc. Med. Image. Comput. Comput. Assist. Interv. (MICCAI), 2011, pp. 58-65.

[37] O. Michailovich, Y. Rathi, and S. Dolui, "Spatially regularized compressed sensing for high angular resolution diffusion imaging," IEEE Trans Med Imaging, vol. 30, pp. 1100-15, 2011. 
[38] B. A. Landman, J. A. Bogovic, H. Wan, F. E. Z. Elshahaby, P.-L Bazin, and J. L. Prince, "Resolution of crossing fibers with constrained compressed sensing using diffusion tensor MRI," Neuroimage, vol. 59, pp. 2175-86, 2012.

[39] S. Merlet, E. Caruyer, and R. Deriche, "Parametric dictionary learning for modeling EAP and ODF in diffusion MRI," in Proc. Med. Image. Comput. Comput. Assist. Interv. (MICCAI), 2012, pp. 10-7.

[40] H. Johansen-Berg and T. Behrens, Diffusion MRI: from quantitative measurement to in-vivo neuroanatomy. London: Academic Press, 2009.

[41] H. E. Assemlal, D. Tschumperlé, L. Brun, and K. Siddiqi, "Recent advances in diffusion MRI modeling: Angular and radial reconstruction," Med Image Anal, vol. 15, pp. 369-96, 2011.

[42] J. P. Haldar and R. M. Leahy, "Linear transforms for Fourier data on the sphere: application to high angular resolution diffusion MRI of the brain," Neurolmage, vol. 71, pp. 233-47, 2013.

[43] T. B. Leergaard, N. S. White, A. de Crespigny, I. Bolstad, H. D'Arceuil, J. G. Bjaalie, and A. M. Dale, "Quantitative histological validation of diffusion mri fiber orientation distributions in the rat brain," PLOS ONE, vol. 5, p. e8595, 2010.

[44] A. K. Seehaus, A. Roebroeck, O. Chiry, D. S. Kim, I. Ronen, H. Bratzke, R. Goebel, and R. A. Galuske, "Histological validation of DW-MRI tractography in human postmortem tissue," Cereb Cortex, vol. 23, pp. 442-50, 2013.

[45] C.-P. Lin, V. J. Wedeen, J.-H. Chen, C. Yao, and W.-Y. I. Tseng, "Validation of diffusion spectrum magnetic resonance imaging with manganeseenhanced rat optic tracts and ex vivo phantoms," NeuroImage, vol. 19, pp. 482-95, 2003.

[46] J. Schmahmann, D. Pandya, R. Wang, G. Dai, H. D'Arceuil, A. de Crespigny, and V. Wedeen, "Association fibre pathways of the brain: parallel observations from diffusion spectrum imaging and autoradiography," Brain, vol. 130, pp. 630-53, 2007.

[47] M. Axer, D. Grässel, M. Kleiner, J. Dammers, T. Dickscheid, J. Reckfort, T. Hütz, B. Eiben, U. Pietrzyk, K. Zilles, and K. Amunts, "Highresolution fiber tract reconstruction in the human brain by means of three-dimensional polarized light imaging," Front Neuroinform, vol. 5, pp. 34-34, 2011.

[48] J. D. Tournier, C. H. Yeh, F. Calamante, K. H. Cho, A. Connelly, and C. P. Lin, "Resolving crossing fibres using constrained spherical deconvolution: validation using diffusion-weighted imaging phantom data," NeuroImage, vol. 42, pp. 617-25, 2008.

[49] C. Poupon, B. Rieul, I. Kezele, M. Perrin, F. Poupon, and J.-F. Mangin, "New diffusion phantoms dedicated to the study and validation of highangular-resolution diffusion imaging (HARDI) models," Magn Reson Med, vol. 60, pp. 1276-83, 2008.

[50] F. B. Laun, L. R. Schad, J. Klein, and B. Stieltjes, "How background noise shifts eigenvectors and increases eigenvalues in DTI," MAGMA, vol. 22, pp. 151-8, 2009.

[51] F. B. Laun, S. Huff, and B. Stieltjes, "On the effects of dephasing due to local gradients in diffusion tensor imaging experiments: relevance for diffusion tensor imaging fiber phantoms," Magn Reson Imaging, vol. 27, pp. 541-8, 2009.

[52] P. Pullens, A. Roebroeck, and R. Goebel, "Ground truth hardware phantoms for validation of diffusion-weighted MRI applications," $J$ Magn Reson Imaging, vol. 32, pp. 482-8, 2010.

[53] K. H. Fritzsche, F. B. Laun, H. P. Meinzer, and B. Stieltjes, "Opportunities and pitfalls in the quantification of fiber integrity: what can we gain from Q-ball imaging?" NeuroImage, vol. 51, pp. 242-51, 2010.

[54] A. Moussavi-Biugui, B. Stieltjes, K. Fritzsche, W. Semmler, and F. B. Laun, "Novel spherical phantoms for Q-ball imaging under in vivo conditions," Magn Reson Med, vol. 65, pp. 190-4, 2011.

[55] F. L. Zhou, P. L. Hubbard, S. J. Eichhorn, and G. J. Parker, "Coaxially electrospun axon-mimicking fibers for diffusion magnetic resonance imaging," ACS Appl Mater Interfaces, vol. 4, pp. 6311-6, 2012.

[56] T. A. Kuder, B. Stieltjes, P. Bachert, W. Semmler, and F. B. Laun, "Advanced fit of the diffusion kurtosis tensor by directional weighting and regularization," Magn Reson Med, vol. 67, pp. 1401-11, 2012.

[57] M. Bach, K. H. Fritzsche, B. Stieltjes, and F. B. Laun, "Investigation of resolution effects using a specialized diffusion tensor phantom," Magn Reson Med, 2013, in press.

[58] O. Soderman and B. Jonsson, "Restricted diffusion in cylindrical geometry," J Magn Reson B, vol. 117, pp. 94-7, 1995.

[59] M. Descoteaux, E. Angelino, S. Fitzgibbons, and R. Deriche, "Regularized, fast, and robust analytical Q-ball imaging," Magn Reson Med, vol. 58, pp. 497-510, 2007.

[60] E. Canales-Rodríguez, L. Melie-García, and Y. Iturria-Medina, "Mathematical description of q-space in spherical coordinates: Exact Q-ball imaging," Magn Reson Med, vol. 61, pp. 1350-67, 2009.
[61] H. Gudbjartsson and S. Patz, "The Rician distribution of noisy MRI data," Magn Reson Med, vol. 34, pp. 910-4, 1995.

[62] A. Ramirez-Manzanares, P. A. Cook, and J. C. Gee, "A Comparison of Methods for Recovering Intra-voxel White Matter Fiber Architecture from Clinical Diffusion Imaging Scans," in Proc. Med. Image. Comput. Comput. Assist. Interv. (MICCAI), 2008, pp. 305-12.

[63] F. Dell'acqua, P. Scifo, G. Rizzo, M. Catani, A. Simmons, G. Scotti, and F. Fazio, "A modified damped richardson-lucy algorithm to reduce isotropic background effects in spherical deconvolution," Neurolmage, vol. 49, pp. 1446-58, 2010.

[64] V. Prčkovska, P. Rodrigues, R. Duits, B. ter Haar Romeny, and A. Vilanova, "Extrapolating fiber crossings from DTI data. Can we gain similar information as HARDI?" in Proc. Workshop on Computational Diffusion MRI, MICCAI, 2010, pp. 26-37.

[65] M. Mani, M. Jacob, A. Guidon, C. Liu, A. Song, V. Magnotta, and J. Zhong, "Acceleration of high angular and spatial resolution diffusion imaging using compressed sensing," in Proc. IEEE Int. Symp. Biomed. Imag. (ISBI): From Nano to Macro, 2012, pp. 326-9.

[66] I. Kezele, M. Descoteaux, C. Poupon, F. Poupon, and J. Mangin, "Spherical wavelet transform for ODF sharpening," Med Image Anal, vol. 14, pp. 332-42, 2010.

[67] Y. Gur, F. Jiao, S. Zhu, and C. Johnson, "White matter structure assessment from reduced HARDI data using low-rank polynomial approximations," in Proc. Workshop on Computational Diffusion MRI, MICCAI, 2012.

[68] E. J. Canales-Rodríguez, Y. Iturria-Medina, Y. Alemán-Gómez, and L. Melie-García, "Deconvolution in diffusion spectrum imaging," NeuroImage, vol. 50, pp. 136-49, 2010.

[69] J.-D. Tournier, F. Calamante, and A. Connelly, "MRtrix: Diffusion tractography in crossing fiber regions," Int J Imaging Syst Technol, vol. 22, pp. 53-66, 2012.

[70] S. N. Sotiropoulos, S. Moeller, S. Jbabdi, J. Xu, J. L. Andersson, E. J. Auerbach, E. Yacoub, D. Feinberg, K. Setsompop, L. L. Wald, T. E. J. Behrens, K. Ugurbil, and C. Lenglet, "Effects of image reconstruction on fibre orientation mapping from multichannel diffusion MRI: Reducing the noise floor using SENSE." Magnetic resonance in medicine : official journal of the Society of Magnetic Resonance in Medicine / Society of Magnetic Resonance in Medicine, Feb. 2013.

[71] S. Aja-Fernandez and A. Tristan-Vega, "A review on statistical noise models for Magnetic Resonance Imaging," LPI, ETSI Telecomunicacion, Universidad de Valladolid, Spain, Tech. Rep., 2013.

[72] J. Wansapura, S. Holland, S. Dunn, and W. Ball, "Nmr relaxation times in the human brain at 3.0 tesla," J. Magn. Reson. Imaging, vol. 9, no. 4, pp. 531-538, 1999. 